\title{
The spatial distributions of cooling gas and intrinsic X-ray absorbing material in cooling flows
}

\author{
S.W. Allen and A.C. Fabian \\ Institute of Astronomy, Madingley Road, Cambridge CB3 OHA
}

August 7, 2021

\begin{abstract}
We present the results from a study of the spatial distributions of cooling gas and intrinsic X-ray absorbing material in a sample of nearby, X-ray bright cooling flow clusters observed with the Position Sensitive Proportional Counter (PSPC) on ROSAT. Our method of analysis employs X-ray colour profiles, formed from ratios of the surface brightness profiles of the clusters in selected energy bands, and an adapted version of the deprojection code of Fabian et al. (1981). We show that all of the cooling flow clusters in our sample exhibit significant central concentrations of cooling gas. At larger radii the clusters appear approximately isothermal. In detail, the spatial distributions and emissivity of the cooling material are shown to be in excellent agreement with the predictions from the deprojection code, and can be used to constrain the ages of the cooling flows. The X-ray colour profiles also indicate substantial levels of intrinsic $\mathrm{X}$-ray absorption in the clusters. The intrinsic absorption increases with decreasing radius, and is confined to the regions occupied by the cooling flows. We explore a range of likely spatial distributions for the absorbing gas and discuss the complexities involved in the measurement of column densities from X-ray data. We show that the application of simple spectral models, in which the intrinsic absorber is treated as a uniform foreground screen, will naturally lead to significant underestimates of the true amounts of absorption. Comparison of our results with previously reported observations made with Einstein Observatory Solid State Spectrometer (White et al. 1991) shows reasonable agreement, but requires that the absorbing material only partially covers the X-ray emitting regions. The masses of absorbing gas in the central (30 arcsec radius) regions of the clusters, calculated under the assumption of solar metallicity in the absorbing material, can be accumulated by the cooling flows on timescales of a few $10^{8}$ years, which are much less than the ages of the flows. This implies that most of the material deposited by the cooling flows in these regions cannot remain in $\mathrm{X}$-ray absorbing gas. The results presented in this paper provide strong support for the standard model of inhomogeneous cooling flows in clusters of galaxies.
\end{abstract}

Key words: galaxies: clusters: general - cooling flows - intergalactic medium $\mathrm{X}$-rays: galaxies

\section{INTRODUCTION}

Clusters of galaxies are luminous X-ray sources. The bulk of the X-ray emission arises from bremsstrahlung and line radiation processes in the IntraCluster Medium (ICM). In the most luminous systems, the $(2-10 \mathrm{keV})$ luminosity exceeds $10^{45} \mathrm{erg} \mathrm{s}^{-1}$ and the emission extends to radii $>2$ Mpc. The total mass of the ICM exceeds that of the galaxies by factors of $1-5$. In the outer regions of clusters $(r \gtrsim 1$ $\mathrm{Mpc})$ the ICM is highly diffuse $\left(n_{e} \lesssim 10^{-4} \mathrm{~cm}^{-3}\right)$ and the cooling time far exceeds the Hubble time $\left(t_{\text {cool }} \gtrsim 10^{11} \mathrm{yr}\right)$. In the central regions of most clusters, however, the density of the ICM rises sharply and the cooling time inferred is significantly less than the Hubble time $\left(t_{\text {cool }} \lesssim 10^{9}\right.$ yr; e.g. Edge, Stewart \& Fabian 1992). In the absence of forces other than thermal pressure and gravity, the cooling of the ICM will lead to a slow net inflow of material towards the cluster centre; a process known as a cooling flow. (See Fabian 1994 for a review of the theory and observations of cooling flows.)

Within the idealized model of a homogeneous cooling flow (in which all of the gas at any particular radius has the same temperature and density), all of the cooling gas flows to the centre of the system where it is 'deposited', having radiated away its thermal energy. However, observations of clusters of galaxies show that cooling flows are not homogeneous. The X-ray surface brightness profiles of clusters, 
Table 1. Observation summary

\begin{tabular}{|c|c|c|c|c|c|}
\hline \multirow[b]{2}{*}{ cluster } & \multirow[b]{2}{*}{$\mathrm{z}$} & \multicolumn{2}{|c|}{ OBSERVATIONS } & \multicolumn{2}{|c|}{ X-RAY CENTROID (J2000) } \\
\hline & & Date & ure $(\mathrm{s})$ & R.A. & Dec. \\
\hline Abell 85 & 0.0518 & 1992 Jul 01 & 10086 & $00^{\mathrm{h}} 41^{\mathrm{m}} 50.5^{\mathrm{s}}$ & $-09^{\circ} 17^{\prime} 58^{\prime \prime}$ \\
\hline Abell 3112 & 0.0746 & 1992 Dec 17 & 7600 & $03^{\mathrm{h}} 17^{\mathrm{m}} 58.4^{\mathrm{s}}$ & $-44^{\circ} 14^{\prime} 27^{\prime \prime}$ \\
\hline Abell 426 & 0.0183 & 1992 Feb 02 & 4787 & $03^{\mathrm{h}} 19^{\mathrm{m}} 48.5^{\mathrm{s}}$ & $41^{\circ} 30^{\prime} 27^{\prime \prime}$ \\
\hline Abell 478 & 0.088 & 1991 Aug 31 & 22139 & $04^{\mathrm{h}} 13^{\mathrm{m}} 24.9^{\mathrm{s}}$ & $10^{\circ} 28^{\prime} 04^{\prime \prime}$ \\
\hline Abell 496 & 0.0320 & 1991 Mar 06 & 8852 & $04^{\mathrm{h}} 33^{\mathrm{m}} 37.9^{\mathrm{s}}$ & $-13^{\circ} 15^{\prime} 43^{\prime \prime}$ \\
\hline Abell 644 & 0.0704 & 1993 Apr 27 & 10285 & $08^{\mathrm{h}} 17^{\mathrm{m}} 25.3^{\mathrm{s}}$ & $-06^{\circ} 49^{\prime} 59^{\prime \prime}$ \\
\hline Hydra A & 0.0522 & 1992 Nov 08 & 18070 & $09^{\mathrm{h}} 18^{\mathrm{m}} 06.2^{\mathrm{s}}$ & $-12^{\circ} 05^{\prime} 41^{\prime \prime}$ \\
\hline Coma & 0.0232 & 1991 Jun 17 & 22108 & $12^{\mathrm{h}} 59^{\mathrm{m}} 46.1^{\mathrm{s}}$ & $27^{\circ} 56^{\prime} 21^{\prime \prime}$ \\
\hline Virgo & 0.0043 & 1992 Dec 17 & 9961 & $12^{\mathrm{h}} 30^{\mathrm{m}} 49.8^{\mathrm{s}}$ & $12^{\circ} 23^{\prime} 32^{\prime \prime}$ \\
\hline Centaurus & 0.0104 & 1994 Jul 05 & 3192 & $12^{\mathrm{h}} 48^{\mathrm{m}} 48.7^{\mathrm{s}}$ & $-41^{\circ} 18^{\prime} 44^{\prime \prime}$ \\
\hline Abell 1795 & 0.0634 & 1992 Jan 04 & 36515 & $13^{\mathrm{h}} 48^{\mathrm{m}} 53.1^{\mathrm{s}}$ & $26^{\circ} 35^{\prime} 34^{\prime \prime}$ \\
\hline Abell 2029 & 0.0767 & 1992 Aug 10 & 12353 & $15^{\mathrm{h}} 10^{\mathrm{m}} 55.7^{\mathrm{s}}$ & $05^{\circ} 44^{\prime} 39^{\prime \prime}$ \\
\hline MKW3s & 0.043 & 1992 Aug 15 & 9802 & $15^{\mathrm{h}} 21^{\mathrm{m}} 51.7^{\mathrm{s}}$ & $07^{\circ} 42^{\prime} 09^{\prime \prime}$ \\
\hline Abell 2199 & 0.0309 & 1993 Jul 25 & 40290 & $16^{\mathrm{h}} 28^{\mathrm{m}} 38.4^{\mathrm{s}}$ & $39^{\circ} 33^{\prime} 00^{\prime \prime}$ \\
\hline Cyg A & 0.057 & 1993 Oct 10 & 9447 & $19^{\mathrm{h}} 59^{\mathrm{m}} 28.4^{\mathrm{s}}$ & $40^{\circ} 43^{\prime} 59^{\prime \prime}$ \\
\hline Sersic 159 & 0.0556 & 1993 May 28 & 13137 & $23^{\mathrm{h}} 13^{\mathrm{m}} 58.3^{\mathrm{s}}$ & $-42^{\circ} 43^{\prime} 35^{\prime \prime}$ \\
\hline Abell 2597 & 0.0824 & 1991 Nov 27 & 7094 & $23^{\mathrm{h}} 25^{\mathrm{m}} 20.0^{\mathrm{s}}$ & $-12^{\circ} 07^{\prime} 31^{\prime \prime}$ \\
\hline Abell 4059 & 0.0478 & 1991 Nov 21 & 5514 & $23^{\mathrm{h}} 57^{\mathrm{m}} 02.0^{\mathrm{s}}$ & $-34^{\circ} 36^{\prime} 23^{\prime \prime}$ \\
\hline
\end{tabular}

Notes: Summary of the PSPC observations. From left to right we list the names, redshifts, observation dates, effective exposure times (after corrections for satellite dead time) and the coordinates of the peaks of the X-ray emission (in the $0.4-2.0 \mathrm{keV}$ band) from the clusters.

although sharply peaked, are not as peaked as they would be for homogeneous flows. Rather than all of the cool gas flowing to the centres of the clusters, the data show that cooled material is deposited throughout the central few tens to hundreds of kpc. Typically the deposition occurs with $\dot{M}(r) \propto r$, where $\dot{M}(r)$ is the integrated mass deposition rate within radius $r$. Spatially-resolved X-ray spectroscopy of clusters also demonstrates the presence of a range of temperature and density phases in the central regions of cooling flow clusters (e.g. Fabian et al. 1994; Fukazawa et al. 1994; Allen, Fabian \& Kneib 1996; Allen et al. 1996b; Fabian et al. 1996). The X-ray spectra and imaging data firmly require that cooling flows are inhomogeneous.

For some years, the primary uncertainty with the standard inhomogeneous model of cooling flows (Fabian 1994) was the fate of the cooled matter (see e.g. Fabian, Nulsen $\&$ Canizares 1991). No identification of the large masses of cooled gas, which should presumably be accumulated by the flows over their lifetimes, was made. However, a study of 21 clusters observed with the Solid State Spectrometer (SSS) on the Einstein Observatory by White et al. (1991) showed that large masses of X-ray absorbing material are common in cooling flows. These authors also showed that significant absorption is not present in non cooling-flow clusters. Spatially-resolved X-ray spectroscopy of the massive cooling flow cluster Abell 478 with ROSAT (Allen et al. 1993) showed that the spatial distribution of the intrinsic absorbing material in this cluster is well-matched to that of the cooling flow. Allen et al. (1993) also demonstrated that the mass of absorbing material is in good agreement with the mass likely to have been accumulated by the cooling flow over its lifetime. The data thus suggest the X-ray absorbing material as a plausible sink for much of the cooled gas deposited by cooling flows. Recent spectral studies of cooling flows with ASCA (Fabian et al. 1994; Fabian et al. 1996) have further confirmed the presence of cooling gas and intrinsic absorbing material in cooling flows. However, to date, the constraints on the spatial distributions of these components remain poor.

In this paper, we present detailed results on the distributions of cool gas and intrinsic X-ray absorbing material in a sample of 17 cooling flow clusters observed with the Position Sensitive Proportional Counter (PSPC) on ROSAT. Our target clusters were selected on the basis of the following criteria; that they be X-ray bright $\left(F_{\mathrm{X}, 2-10}>\right.$ $1.7 \times 10^{-11} \mathrm{erg} \mathrm{cm}^{-2} \mathrm{~s}^{-1}$; Edge et al. 1990), relatively nearby $(z<0.1$; allowing them to be resolved on scales of tens of kpc with the PSPC), and contain large cooling flows $\left(\dot{M}>100 \mathrm{M}_{\odot} \mathrm{yr}^{-1}\right)$. We also include two lower-luminosity (smaller $\dot{M}$ ) systems, the Centaurus cluster and the Virgo cluster, since they are previously well-studied and since good $\mathrm{X}$-ray data exist for them. Finally, we included one noncooling flow cluster, the Coma cluster, in our sample as a control.

The large intrinsic column densities $\left(\Delta N_{\mathrm{H}} \sim 10^{21}\right.$ atom $\mathrm{cm}^{-2}$ ) in cooling flows inferred from the Einstein SSS (White et al. 1991) and ASCA data (Fabian et al. 1994) must have a significant effect on the flux detected with the PSPC at energies below about $0.8 \mathrm{keV}$. Indeed, few X-rays can escape the absorbed region at energies below the carbon edge in the detector window at $0.28 \mathrm{keV}$. However, this does not mean that no X-rays escape the cluster in the carbon band, since there is always a large contribution from the outer, less intrinsically absorbed, parts of the cluster along any 
line of sight. Given the spectral resolution of the PSPC $\left[\Delta E / E=0.43(E / 0.93 \mathrm{keV})^{-0.5}\right]$ and the energy-dependent effects of absorption, one might expect that the lowest energy range observable with the PSPC (0.1-0.4 keV) would be optimum for absorption studies. Unfortunately, however, the spatial resolution of the PSPC is substantially worse in this band than at higher energies. A significant fraction of the counts from a point source are scattered to radii $>1$ arcmin in the $0.1-0.2 \mathrm{keV}$ band, and in the $0.2-0.4 \mathrm{keV}$ band the Point-Spread-Function (PSF) is still almost a factor of $\sim 2$ larger than at higher $(0.4-2.0 \mathrm{keV})$ energies (Hasinger et al. 1992). In order to make meaningful spectra, the spatial regions selected must be at least as large as the largest relevant PSF. Since bright cooling flows typically have radii of only one or two arcmin, it is then difficult to make spatiallyresolved studies of cooling flows with the PSPC using data from the softest band. Certainly, any study of the distribution of absorbing matter from such an exercise is very limited.

The problems with the PSF are compounded by the large number of parameters required to model the spectrum of an absorbed cooling flow, and the small number of emission-independent spectral bands of the PSPC. Even a simple model for an absorbed cooling flow involves 5 free parameters; the normalization, abundance and temperature of the ambient cluster gas, the cooling rate in the cluster and the column density of intrinsic absorption. (This assumes that cluster redshift and Galactic column density are known beforehand.) Although the counts in each of the 256 PSPC spectral channels are statistically independent, the limited spectral resolution of the detector means that there are only about 5 independent spectral bands from a modelling point of view. Consequently, most spectral analyses with ROSAT data employ simple models of gas at a single temperature and with a uniform screen of absorption. We term these 'single-phase' models. [Temperatures and column densities determined with single-phase models should only be interpreted as some emission/detector-weighted mean over a range of temperatures or column densities at any projected radius.] Only with the much higher spectral resolution of instruments such as the Einstein Observatory SSS and the ASCA Solid-state Imaging Spectrometers (SIS) can more detailed spectral fits be attempted.

In this paper we make use of X-ray colour profiles, rather than individual spectra, to study the spatial distributions of cool gas and absorbing material in clusters. By restricting our study to the $0.4-2.0 \mathrm{keV}$ band, where the FWHM of the PSF is approximately constant at 25 arcsec, we can make optimum use of the spatial resolution of the PSPC. Emission lines, principally due to Fe-L transitions characteristic of gas cooling through temperatures of $\sim 1 \mathrm{keV}$, are strongest between energies of 0.8 and $1.4 \mathrm{keV}$. Intrinsic column densities at the level of $\sim 10^{21}$ atom $\mathrm{cm}^{-2}$ should have a marked effect on the spectrum below about $0.8 \mathrm{keV}$. The remaining $1.4-2.0 \mathrm{keV}$ band is comparatively little-affected by emission lines and absorption. We have therefore divided the $0.4-2.0 \mathrm{keV}$ band into bands $\mathrm{B}$, covering the range $0.41-0.79 \mathrm{keV}$, C from 0.80 $1.39 \mathrm{keV}$ and D from $1.4-2.0 \mathrm{keV}$. The ratio of B to D should be sensitive to absorption, and the ratio of $\mathrm{C}$ to $\mathrm{D}$ to the presence of cool gas (where 'cool' implies a temperature significantly less than that of the bulk of the ICM). By creat-

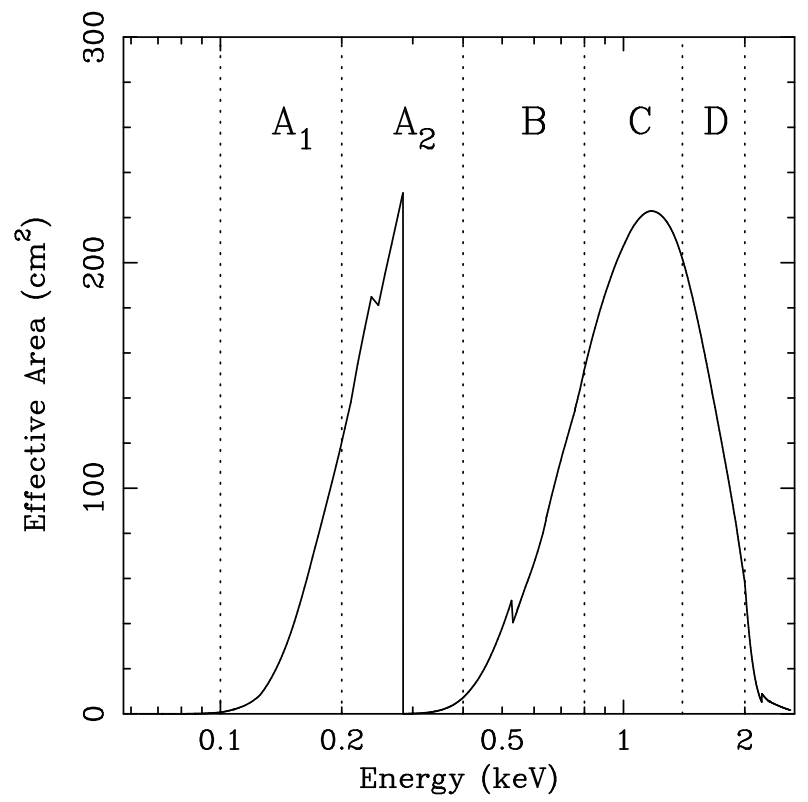

Figure 1. Representation of the energy bands used in the X-ray colour profile analysis, overlaid on the effective area curve for the PSPC.

ing ratios of the surface brightness profiles of the clusters in these bands, we parameterize the amounts of cool gas and absorbing material in the clusters and, to the limits of the spatial resolution of the PSPC, constrain the spatial distributions of these components. We then compare these results to the predictions from standard cooling flow models.

The structure of the paper is as follows. In Section 2 we summarize the observations and data reduction. In Section 3 we present the results from a standard deprojection analysis of the data. In Section 4 we present the results on the X-ray colour profiles and relate them to expectations from simulated cluster spectra. In Section 5 we compare the observed colour profiles to the predictions from the deprojection analysis and determine the results on the distributions of cool gas and X-ray absorbing material in the clusters. In Section 6 we discuss the complexities associated with the measurement of intrinsic column densities with PSPC data. Section 7 summarizes our conclusions. Throughout this paper, $H_{0}=50$ $\mathrm{kms}^{-1} \mathrm{Mpc}^{-1}, \Omega=1$ and $\Lambda=0$ are assumed.

\section{OBSERVATIONS}

The PSPC observations were carried out between 1991 March 6 and 1994 July 5. Exposure times range from 3192s for the Centaurus cluster to 40290s for Abell 2199. Details of the individual cluster observations are summarized in Table 1. In cases where more than one observation of a source was made, only the longest of the individual exposures has been used. This minimizes systematic errors in the central surface brightness profiles that could be introduced by mosaicing. For the Coma cluster, for which four individual observations were made at different pointing positions, we have used the 
Table 2. Definition of the energy bands

\begin{tabular}{cc} 
Band & Energy range $(\mathrm{keV})$ \\
\hline & \\
$A_{1}$ & $0.10-0.40$ \\
$A_{2}$ & $0.20-0.40$ \\
$\mathrm{~B}$ & $0.41-0.79$ \\
$\mathrm{C}$ & $0.80-1.39$ \\
$\mathrm{D}$ & $1.40-2.00$ \\
$\mathrm{~F}$ & $0.41-2.00$
\end{tabular}

data set that places the peak of the cluster emission closest to the centre of the PSPC field of view. For the Centaurus cluster, we have used only the data from the $3.2 \mathrm{ks}$ observation in 1994 July, which was less-affected by scattered solar $\mathrm{X}$-ray emission than earlier observations.

The data were reduced using the Starlink ASTERIX package. X-ray images with $15 \times 15 \operatorname{arcsec}^{2}$ pixels were extracted in each of the energy bands listed in Table 2 . Regions affected by emission from point sources were masked out and ignored. Corrections for telescope vignetting were made and the data were background subtracted using circular regions of radius 6 arcmin, offset by $\sim 40$ arcmin from the centres of the fields (except in the case of the Virgo cluster, where a 3 arcmin radius region offset by 45 arcmin was used). Accurate centroids for the $\mathrm{X}$-ray emission were determined from the full $0.4-2.0 \mathrm{keV}$ (Band F) images. These are also listed in Table 1. X-ray surface brightness profiles were then extracted, about these centroids, in each energy band.

\section{DEPROJECTION ANALYSIS}

The $\mathrm{F}$ band $(0.4-2.0 \mathrm{keV})$ images of the clusters have been analysed using an updated version of the deprojection code of Fabian et al. (1981; see also Thomas, Fabian \& Nulsen 1987). Under assumptions of spherical symmetry and hydrostatic equilibrium in the ICM, the deprojection technique can be used to study the properties of the intracluster gas (e.g. density, pressure, temperature, cooling rate) as a function of radius. The deprojection method requires that either the total mass profile (which defines the pressure profile) or the gas temperature profile be specified. Following ASCA observations of a number of the clusters included in the sample (Fabian et al. 1996; Ohashi et al. 1996), and the results from the combined X-ray and gravitational lensing study of the cooling-flow cluster PKS0745-191 by Allen et al. (1996a), we assume that the mass-weighted temperature profiles in the clusters remain approximately constant, at the temperatures listed in Table 3. Note that the assumption of a constant mass-weighted temperature profile is consistent with ROSAT results which often show a drop in the emission-weighted temperatures in the central regions of nearby cooling-flow clusters. Such measurements will naturally result from the presence of high emissivity cooling gas, distributed throughout the (inhomogeneous) cooling flow with $\dot{M} \underset{\sim}{\sim}$, even when the mass-weighted temperature pro-
Table 4. A comparison of isothermal and cooling gas

\begin{tabular}{ccccc} 
& \multicolumn{2}{c}{ Isothermal } & \multicolumn{2}{c}{ C. Flow } \\
$k T(\mathrm{keV})$ & C/D & B/D & C/D & B/D \\
\hline & & & & \\
1 & 5.30 & 2.08 & 7.76 & 4.76 \\
2 & 3.27 & 1.61 & 4.72 & 2.60 \\
3 & 2.97 & 1.51 & 4.02 & 2.21 \\
4 & 2.83 & 1.44 & 3.71 & 2.02 \\
5 & 2.74 & 1.39 & 3.53 & 1.88 \\
6 & 2.68 & 1.36 & 3.40 & 1.79 \\
7 & 2.64 & 1.34 & 3.30 & 1.73 \\
8 & 2.60 & 1.32 & 3.22 & 1.68 \\
9 & 2.58 & 1.30 & 3.15 & 1.66 \\
10 & 2.56 & 1.29 & 3.11 & 1.63
\end{tabular}

Notes: A comparison of the theoretical $\mathrm{C} / \mathrm{D}$ and $\mathrm{B} / \mathrm{D}$ ratios for isothermal gas and a constant pressure cooling flow. For the cooling flow data, the $k T$ value is the ambient cluster temperature from which the gas cools. A Galactic column density of $10^{20}$ atom $\mathrm{cm}^{-2}$ and a metallicity of $0.5 Z_{\odot}$ are assumed.

file of the inflowing material remains constant (e.g. Allen et al. 1996a,b).

The primary results on the cooling flows in the clusters; the cooling times in the central 30 arcsec bin, the cooling radii (the radii at which the cooling time first exceeds the Hubble time) and the integrated mass deposition rates within the cooling radii, are listed in Table 3. In all cases the mass deposition from the cooling flows is distributed throughout the cooling radii of the clusters, with $\dot{M} \underset{\sim}{\sim}$.

\section{THE X-RAY COLOUR PROFILES}

As discussed in Section 1, our method of analysis makes use of X-ray colour profiles, rather than the more standard approach of taking annular or regional spectra, to study the distributions of cool gas and intrinsic absorbing material in the clusters. Such a technique allows us to make the best use of the spatial resolution offered by the ROSAT PSPC.

We have constructed radial surface brightness profiles for the clusters in each the energy bands, B, C and D (Table 2 ). The surface brightness profiles were binned to a spatial scale of 30 arcsec pixel $^{-1}$ (slightly larger than the FWHM resolution of the PSPC) which provides a good signal-tonoise ratio in the pixels over the regions of interest. Figs. 2 and 3 summarize the results on the X-ray colour profiles. Fig. 2 shows the $\mathrm{C} / \mathrm{D}$ profiles, which are approximately constant at large radii but increase in their central regions. The B/D profiles, shown in Fig. 3, are generally flatter than the $\mathrm{C} / \mathrm{D}$ data.

Figs. 4 and 5 illustrate how the X-ray colour profiles may be used to probe the physical properties of the clusters. These figures show the behaviour of the $\mathrm{C} / \mathrm{D}$ and $\mathrm{B} / \mathrm{D}$ ratios in simulated cluster spectra as a function of temperature, column density and metallicity. Results are shown both for isothermal gas and for gas cooling at constant pressure, from the ambient cluster temperature to a temperature below the ROSAT band i.e. a constant pressure cooling flow. The sim- 
Table 3. Cooling flow results

\begin{tabular}{|c|c|c|c|c|c|c|}
\hline & $\begin{array}{c}k T \\
(\mathrm{keV})\end{array}$ & $\begin{array}{c}N_{\mathrm{H}} \\
\left(10^{20} \text { atom } \mathrm{cm}^{-2}\right)\end{array}$ & $\begin{array}{l}r_{\mathrm{cool}} \\
(\mathrm{kpc})\end{array}$ & $\begin{array}{c}r_{\text {cool }} \\
(\operatorname{arcmin})\end{array}$ & $\begin{array}{c}t_{\text {cool }} \\
\left(10^{9} \text { yr }\right)\end{array}$ & $\begin{array}{c}\dot{M} \\
\left(\mathrm{M}_{\odot} \mathrm{yr}^{-1}\right)\end{array}$ \\
\hline Abell 85 & $6.6_{-1.4}^{+1.8}$ & 2.96 & $137_{-34}^{+8}$ & $1.66_{-0.41}^{+0.10}$ & $2.85_{-0.11}^{+0.15}$ & $187_{-42}^{+16}$ \\
\hline Abell 3112 & $4.1_{-1.1}^{+\frac{1}{2} .4}$ & 2.78 & $251_{-46}^{+74}$ & $2.19_{-0.40}^{+0.41}$ & $2.13_{-0.08}^{+0.11}$ & $406_{-57}^{+42}$ \\
\hline Abell 426 & $6.3_{-0.2}^{+0.2}$ & 13.7 & $\begin{array}{l}169_{-6}^{+9} \\
+9\end{array}$ & $\begin{array}{l}5.46_{-0.19}^{+0.20} \\
\end{array}$ & $1.06_{-0.05}^{+0.06}$ & $456_{-19}^{+16}$ \\
\hline Abell 478 & $7.0_{-0.5}^{+0.2}$ & 20.0 & $213_{-48}^{+19}$ & $1.61_{-0.36}^{+0.19}$ & $2.59_{-0.06}^{+0.05}$ & $717_{-107}^{-199}$ \\
\hline Abell 496 & $4.8_{-0.8}^{+0.9}$ & 4.27 & $105_{-13}^{+14}$ & $1.99_{-0.25}^{+0.26}$ & $1.84_{-0.09}^{+0.08}$ & $85_{-9}^{+10}$ \\
\hline Abell 644 & $8.1_{-2.5}^{+2.6}$ & 8.61 & $200_{-61}^{+50}$ & $1.83_{-0.56}^{+0.25}$ & $8.28_{-0.80}^{+1.27}$ & $244_{-93}^{+69}$ \\
\hline Hydra A & $3.6_{-0.5}^{+0.5}$ & 4.84 & $161_{-15}^{+61}$ & $1.93_{-0.18}^{+0.56}$ & $1.63^{+0.03}$ & $\begin{array}{l}267^{+93} \\
+48\end{array}$ \\
\hline Coma & $8.0_{-0.3}^{+0.3}$ & 0.90 & $0_{-0}^{+29}$ & $0_{-0}^{+0.75}$ & $18.4_{-5.9}^{+0.04}$ & $0_{-0}^{+1}$ \\
\hline Virgo & $2.4_{-0.3}^{-0.3}$ & 1.71 & $\begin{array}{c}-0 \\
98_{-2}^{+28}\end{array}$ & $13.16_{-0}^{+3.76}$ & $0.21_{-0.5}^{+0.91}$ & $\begin{array}{c}-0 \\
37_{-6}^{+28}\end{array}$ \\
\hline Centaurus & $3.6_{-0.4}^{+0.3}$ & 8.87 & $87_{-29}^{+-2}$ & $4.88_{-1.63}^{+0.39}$ & $0.70_{-0.04}^{+0.01}$ & $\begin{array}{r}-6 \\
27_{-7}^{+5}\end{array}$ \\
\hline Abell 1795 & $5.1_{-0.5}^{+0.4}$ & 1.11 & $192_{-18}^{+39}$ & $1.93_{-0.18}^{+0.32}$ & $2.14_{-0.04}^{+0.04}$ & $447_{-30}^{+50}$ \\
\hline Abell 2029 & $7.1_{-1.4}^{+2.5}$ & 3.05 & $200_{-53}^{+\frac{1}{+6}}$ & $1.70_{-0.45}^{+0.18}$ & $2.76_{-0.08}^{+0.04}$ & $590_{-96}^{+30}$ \\
\hline MKW3s & $2.8_{-0.4}^{-1.4}$ & 2.86 & $154_{-32}^{+73}$ & $2.21_{-0.46}^{+1.05}$ & $2.17_{-0.17}^{+0.08}$ & $161_{-29}^{-96}$ \\
\hline Abell 2199 & $4.7_{-0.4}^{-0.4}$ & 0.87 & $142_{-27}^{+24}$ & $2.78_{-0.53}^{+0.46}$ & $1.94_{-0.04}^{+0.17}$ & $162_{-30}^{+29}$ \\
\hline Cygnus A & $4.1_{-1.3}^{+4.3}$ & 32.8 & $157_{-44}^{+46}$ & $1.74_{-0.49}^{+0.53}$ & $1.39_{-0.05}^{+0.04}$ & $320_{-32}^{+30}$ \\
\hline Sersic 159 & $2.9_{-0.8}^{+1.3}$ & 1.80 & $167_{-11}^{-33}$ & $1.89_{-0.12}^{+0.49}$ & $1.75_{-0.06}^{+0.06}$ & $231_{-10}^{+11}$ \\
\hline Abell 2597 & $\begin{array}{l}-0.8 \\
>3.8\end{array}$ & 2.46 & $162_{-6}^{+57}$ & $1.29_{-0.05}^{+0.12}$ & $2.44_{-0.07}^{+0.11}$ & $299_{-17}^{+10}$ \\
\hline Abell 4059 & $3.5_{-0.6}^{+0.6}$ & 1.61 & $134_{-38}^{+-67}$ & $1.74_{-0.49}^{+1.00}$ & $3.53_{-0.48}^{+0.06}$ & $115_{-37}^{+57}$ \\
\hline
\end{tabular}

Notes: A summary of the results from the deprojection analyses of the F band images. Temperature $(k T)$ data are from Edge et al (1990) except for Abell 426 (Allen et al. 1992) and Abell 478 (Allen et al. 1993). $N_{\mathrm{H}}$ values are Galactic column densities from Stark et al. (1992) except for Abell 478 which is from Johnstone et al. (1992). Cooling radii $\left(r_{\text {cool }}\right)$ are quoted in both kpc and arcmin. Cooling times $\left(t_{\text {cool }}\right)$ for the central (30 arcsec radius) bin are in $10^{9} \mathrm{yr}$, and mass deposition rates $(\dot{M})$ in $\mathrm{M}_{\odot} \mathrm{yr}^{-1}$. Errors on the cooling times are the 10 and 90 percentile values from 100 Monte Carlo simulations. The upper and lower confidence limits on the cooling radii are the points where the 10 and 90 percentiles exceed and become less than the Hubble time, respectively. Errors on the mass deposition rates are the 90 and 10 percentile values at the upper and lower limits for the cooling radius.

ulated spectra were constructed using the plasma code of Kaastra \& Mewe (1993) and the photoelectric absorption models of Morrison \& McCammon (1983). We adopt the definition of solar abundance from Anders \& Grevesse (1989). In all cases the absorbing column density is modelled as a uniform screen, with solar metallicity, at zero redshift. A cluster redshift of $z=0.01$ is assumed (although the results do not vary significantly for any redshift $\leq 0.1$ ).

The theoretical curves for an isothermal plasma are shown in Figs. 4(a)-(d). At high temperatures $(k T \gtrsim 3 \mathrm{keV})$ both the $\mathrm{C} / \mathrm{D}$ and $\mathrm{B} / \mathrm{D}$ ratios are weak functions of temperature. At lower temperatures, however, the $\mathrm{C} / \mathrm{D}$ ratio (in particular) increases sharply as the temperature drops, due primarily to $\mathrm{Fe}-\mathrm{L}$ emission lines in the $\mathrm{C}$ band produced by gas at $\sim 1 \mathrm{keV}$. Both the B/D and C/D ratios decrease with increasing column density, although the variation of the $\mathrm{B} / \mathrm{D}$ ratio is steeper (particularly at low column densities). Both ratios also exhibit some variation with metallicity. In general, as the metallicity rises the $\mathrm{C} / \mathrm{D}$ ratio increases (slightly) and the $\mathrm{B} / \mathrm{D}$ ratio drops, although for $k T \gtrsim 3 \mathrm{keV}$ the functions are very weak.

The behaviour for cooling gas, shown in Figs. 5(a)-(d), is qualitatively much like that for isothermal emission, except that the $\mathrm{C} / \mathrm{D}$ and $\mathrm{B} / \mathrm{D}$ ratios generally give higher values for the same temperature, column density and metallicity. This is illustrated by the data presented in Table 4 where we list the $\mathrm{C} / \mathrm{D}$ and $\mathrm{B} / \mathrm{D}$ values for isothermal and cooling plasmas as a function of temperature, for a fixed column density of $10^{20}$ atom $\mathrm{cm}^{-2}$ and a metallicity of $0.5 \mathrm{Z}_{\odot}$. The variation of B/D with metallicity, Fig. $5(\mathrm{~d})$, is flatter for cooling gas than for an isothermal plasma, and exhibits a downwards trend at low metallicities not seen in the isothermal data.

In what follows, we use the $\mathrm{C} / \mathrm{D}$ profiles to demonstrate the presence of distributed cooling gas in the central regions of the cooling flow clusters, and the B/D ratio to demonstrate the presence of intrinsic X-ray absorbing gas.

\section{RESULTS FROM THE COLOUR PROFILES}

\section{$5.1 \mathrm{C} / \mathrm{D}$ and cooling radii}

The C/D colour profiles shown in Fig. 2 exhibit a number of striking features. Firstly, we see that at large radii $\left(r>r_{\text {cool }}\right)$ the profiles are essentially flat. The observed values are generally in good agreement with the theoretical predictions for isothermal gas at the temperatures listed in Table 3, absorbed by the appropriate Galactic column density (although significant deviations are observed for Abell 478, the Coma cluster, and Abell 2199, the origins of which are discussed in Section 5.3.) The observed and predicted $\mathrm{C} / \mathrm{D}$ and $\mathrm{B} / \mathrm{D}$ ratios at large radii are summarized in Table 5 . 

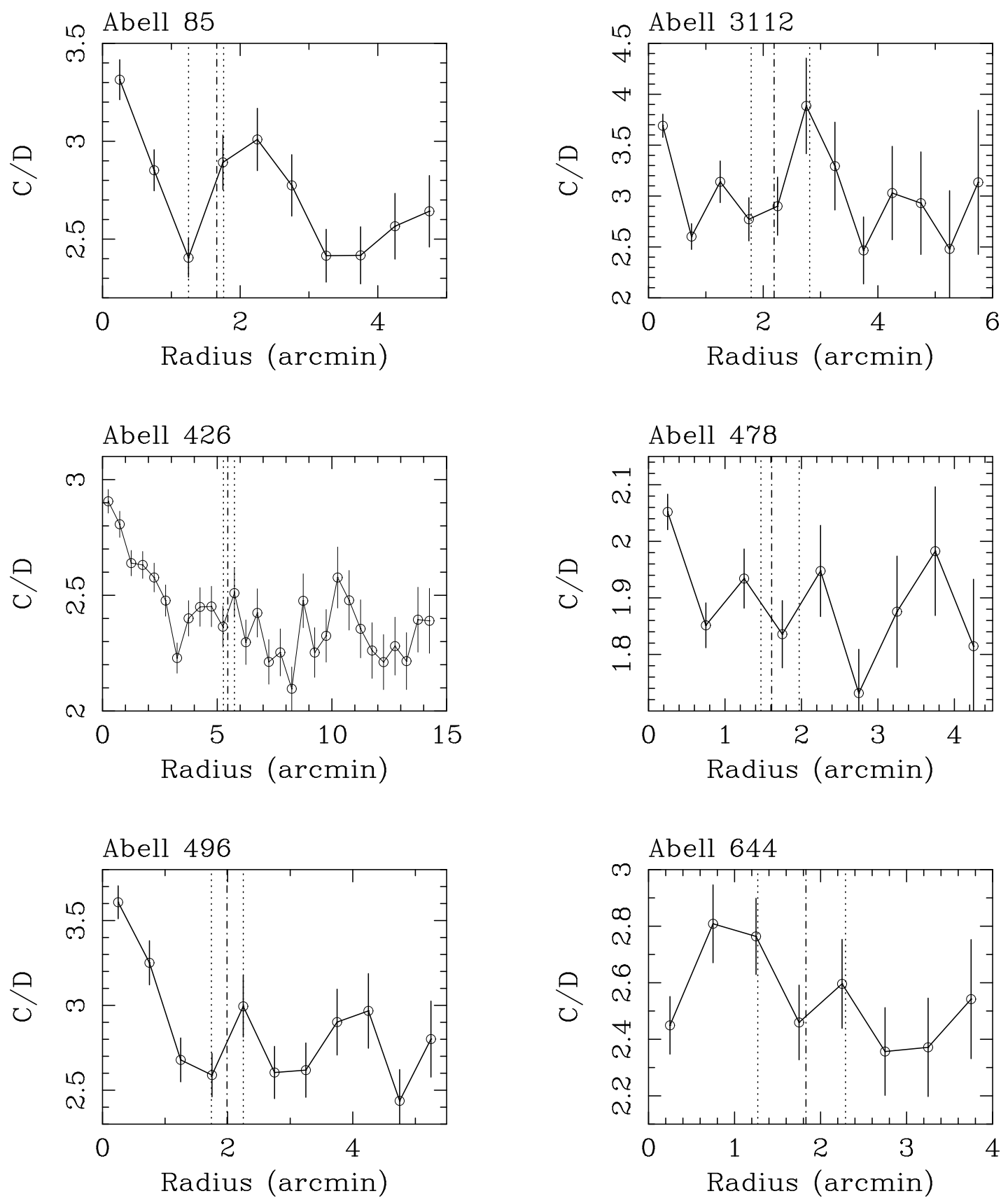

Figure 2. The C/D $(0.80-1.39 \mathrm{keV} / 1.40-2.00 \mathrm{keV}) \mathrm{X}$-ray colour profiles (with $1 \sigma$ error bars). The cooling radii determined from the deprojection analysis (Section 3) are indicated by the vertical dot-dashed lines. The 10 and 90 per cent confidence ranges on those radii are marked by the dotted lines. The profiles are essentially flat at large radii but show clear increases within $r_{\text {cool }}$.

Within radii $r \lesssim r_{\text {cool }}$, however, the cooling-flow clusters exhibit clear increases in their $\mathrm{C} / \mathrm{D}$ ratios. From comparison with the theoretical curves in Figs. 4 and 5, we see that these increases can only plausibly be explained by the presence of cooler gas. The X-ray colour profiles thus provide firm evidence for distributed cool(ing) gas in the central regions of the cooling flow clusters and show that this gas is confined within the cooling radii inferred from the deprojection analyses. For the one non-cooling flow cluster in the sample, the Coma cluster, no significant enhancement in the $\mathrm{C} / \mathrm{D}$ ratio (outside the central $30 \operatorname{arcsec}$ i.e. $20 \mathrm{kpc}$ ) is observed.

\subsection{The spatial distribution of cooling gas}

The $\mathrm{C} / \mathrm{D}$ profiles shown in Fig. 2 clearly demonstrate the presence of distributed cool gas in the centres of the cooling flow clusters. The regions over which the cool gas is 

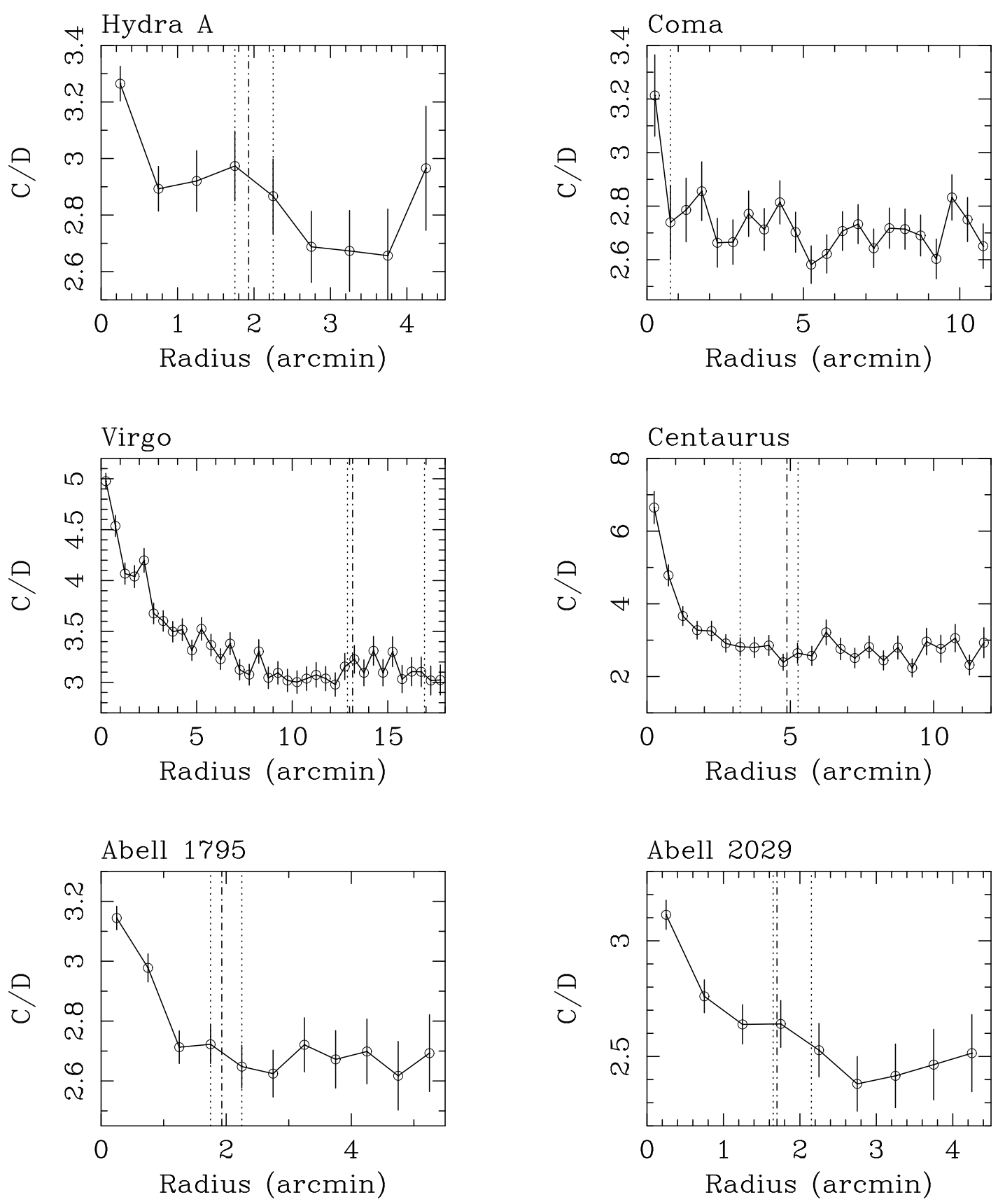

Figure 2. - continued

distributed are well matched to the cooling radii inferred from the deprojection analysis (Section 3). Given this agreement, we have attempted a more sophisticated comparison of the $\mathrm{C} / \mathrm{D}$ profiles with the predictions from the deprojection code.

The deprojection analysis employed in Section 3 divides the X-ray emission from the cluster into a series of concentric spherical shells. The luminosity in shell $j$ may be written as the sum of four components.
$L_{j}=\Delta \dot{M}_{j} H_{j}+\Delta \dot{M}_{j} \Delta \Phi_{j}+\left[\sum_{i=1}^{j-1} \Delta \dot{M}_{i}\left(\Delta \Phi_{j}+\Delta H_{j}\right)\right]$,

where $\Delta \dot{M}_{j}$ is the mass deposited in shell $j, H_{j}$ is the enthalpy of the gas in shell $j$, and $\Delta \Phi_{j}$ is the gravitational energy released in crossing the shell. $\sum_{i=1}^{j-1} \Delta \dot{M}_{i}$ is the mass flow rate through shell $j$, and $\Delta H_{j}$ the change of enthalpy of the gas as it moves through the shell.

Thus the first term in equation 1 accounts for the enthalpy of the gas deposited in shell $j$. The second term is the gravitational work done on the gas deposited in shell $j$. The 

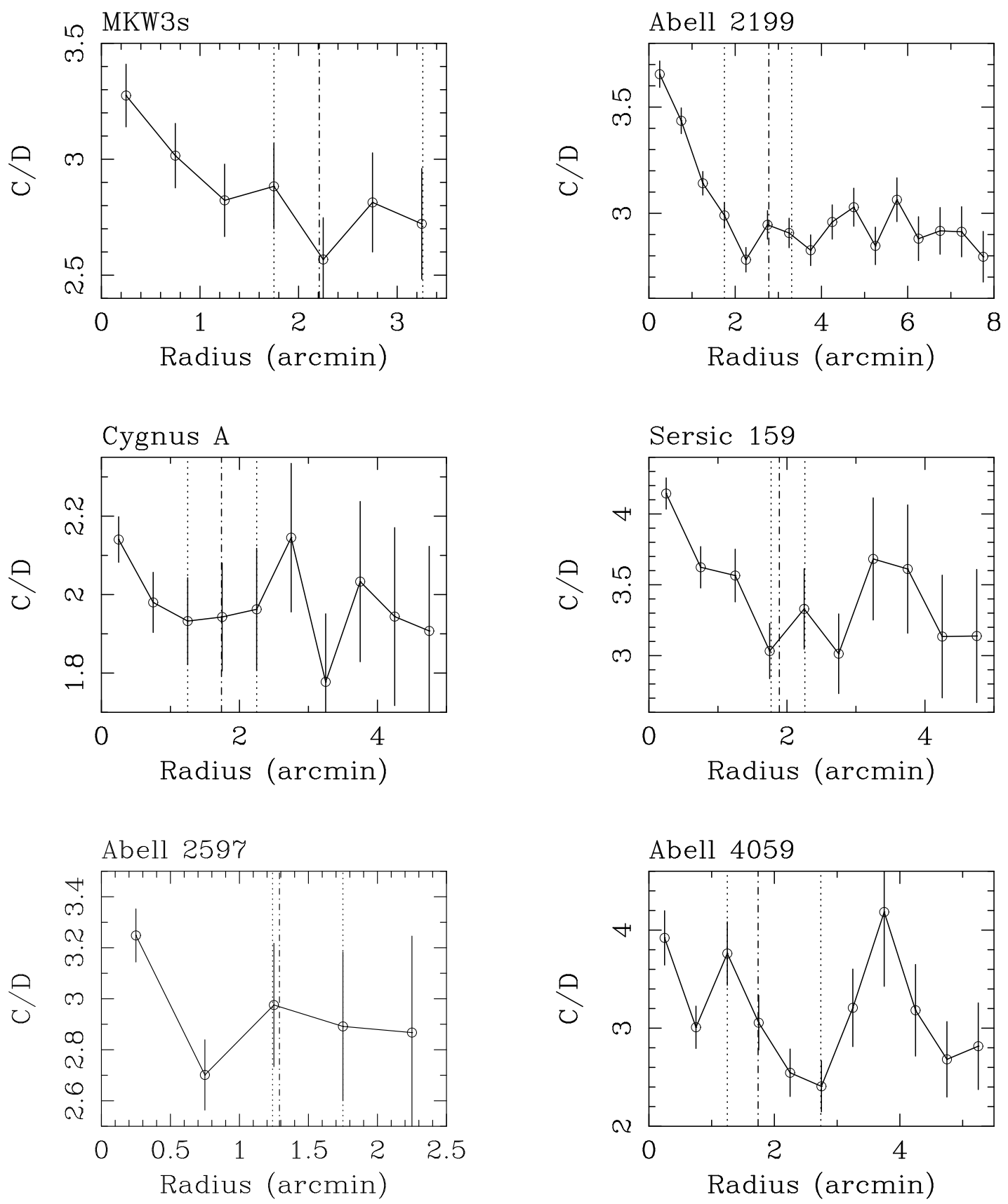

Figure 2. - continued

third and fourth terms account for the gravitational work done on gas flowing through shell $j$ to interior radii, and the enthalpy released by that gas as it passes through the shell. Since we have constrained the mass-weighted temperatures in the cooling flows to remain approximately constant with radius (at the values listed in Table 3 ) the luminosity in the fourth term is negligible.

The luminosity contributed by the second and third terms will have a spectrum that can be approximated by an isothermal plasma at the appropriate temperature for the cluster ( $c f$. Fig. 4). The emission accounted for in the first term, however, should have the spectrum appropriate for gas cooling from the ambient cluster temperature ( $c f$. Fig. 5). By assigning the appropriate $\mathrm{C} / \mathrm{D}$ values from the theoretical curves, corrected for redshift effects, to each of the terms in equation 1 , the deprojection code can be used to predict intrinsic and projected $\mathrm{C} / \mathrm{D}$ colour profiles for the clusters. These predictions can then be compared to the observed values.

In Fig. 6 we show the comparison of the observed and predicted $\mathrm{C} / \mathrm{D}$ profiles for the cooling flows in the sample. Due to the finite ages of the clusters, we limit the comparison to those radii where the cooling time of the cluster gas is $\leq 5 \times 10^{9} \mathrm{yr}$. In the outer regions of the clusters the emis- 

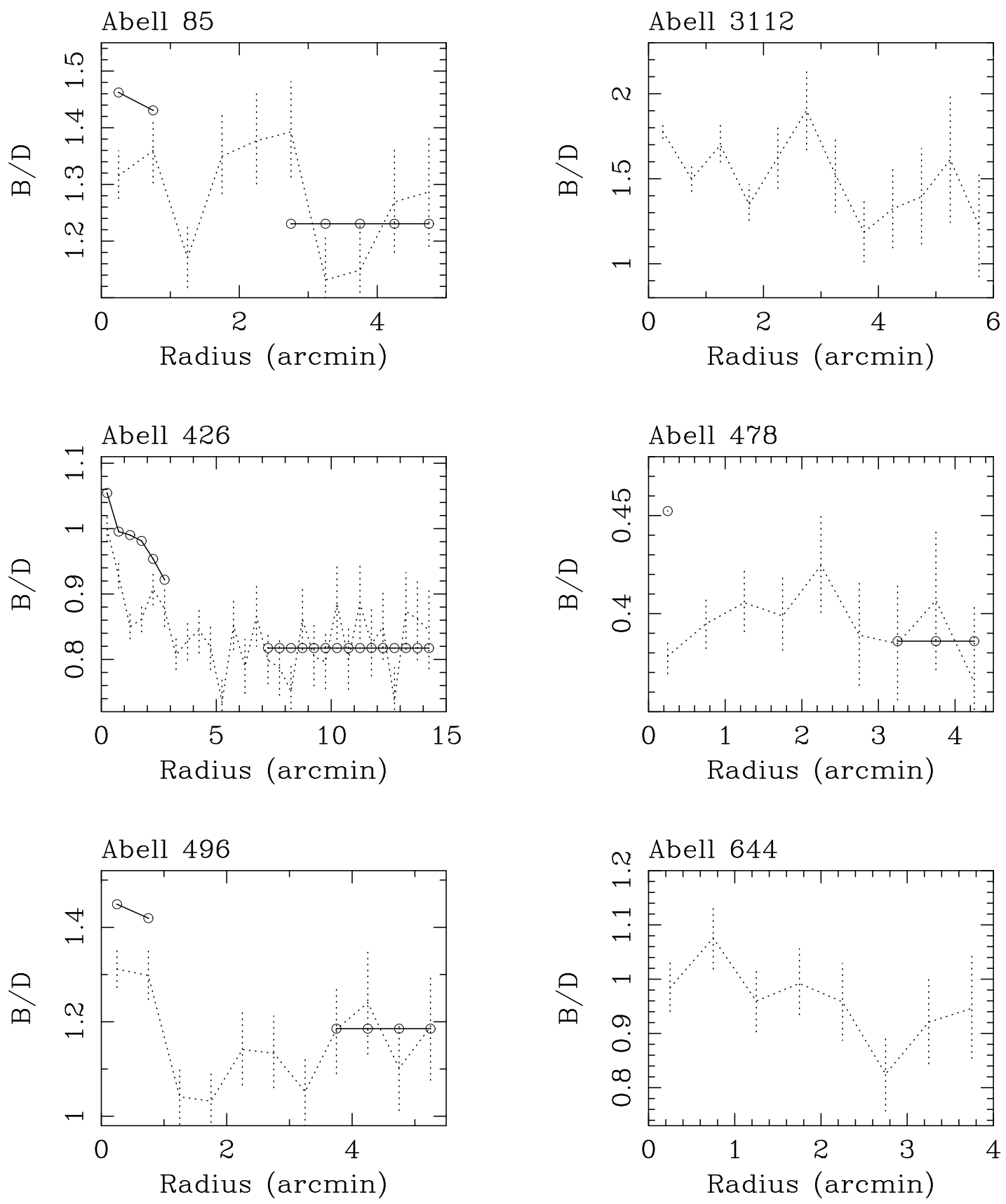

Figure 3. The B/D $(0.40-0.79 \mathrm{keV} / 0.80-1.39 \mathrm{keV})$ X-ray colour profiles (with $1 \sigma$ error bars). Circled points mark the values predicted from the deprojection code (Section 5.3) assuming Galactic absorption. These predicted values are only plotted for radii where the cooling time of the gas is less than $5 \times 10^{9}$ yr. Note the clear deficit in the observed B/D values relative to the predictions from the deprojection code in the central regions, which indicates the need for excess absorption. In the outer regions the circled points mark the best-fitting constant $\mathrm{B} / \mathrm{D}$ value (as listed in Table 5).

sion is assumed to be isothermal, at the temperature given in Table 3, and absorbed by the appropriate Galactic column density. The predicted curves are then re-normalized to match the observed $\mathrm{C} / \mathrm{D}$ values in the outer regions of the clusters. (Note that the normalization factors, which are given by the ratios of the observed to predicted $\mathrm{C} / \mathrm{D}$ ratios in the outer regions of the clusters, are typically close to unity.) We do not attempt any comparison for either the
Centaurus or Virgo clusters since both systems are relatively cool and are known to contain strong metallicity gradients (Allen \& Fabian 1994; Fukazawa et al. 1994). The low redshifts of these systems also means that some drop in their mass-weighted temperatures, as the gravitational potentials of the clusters merge with those of the central galaxies, are likely in the innermost 1-2 bins. Indeed, such a temperature drop is required to explain the large $\mathrm{C} / \mathrm{D}$ ratio observed in 

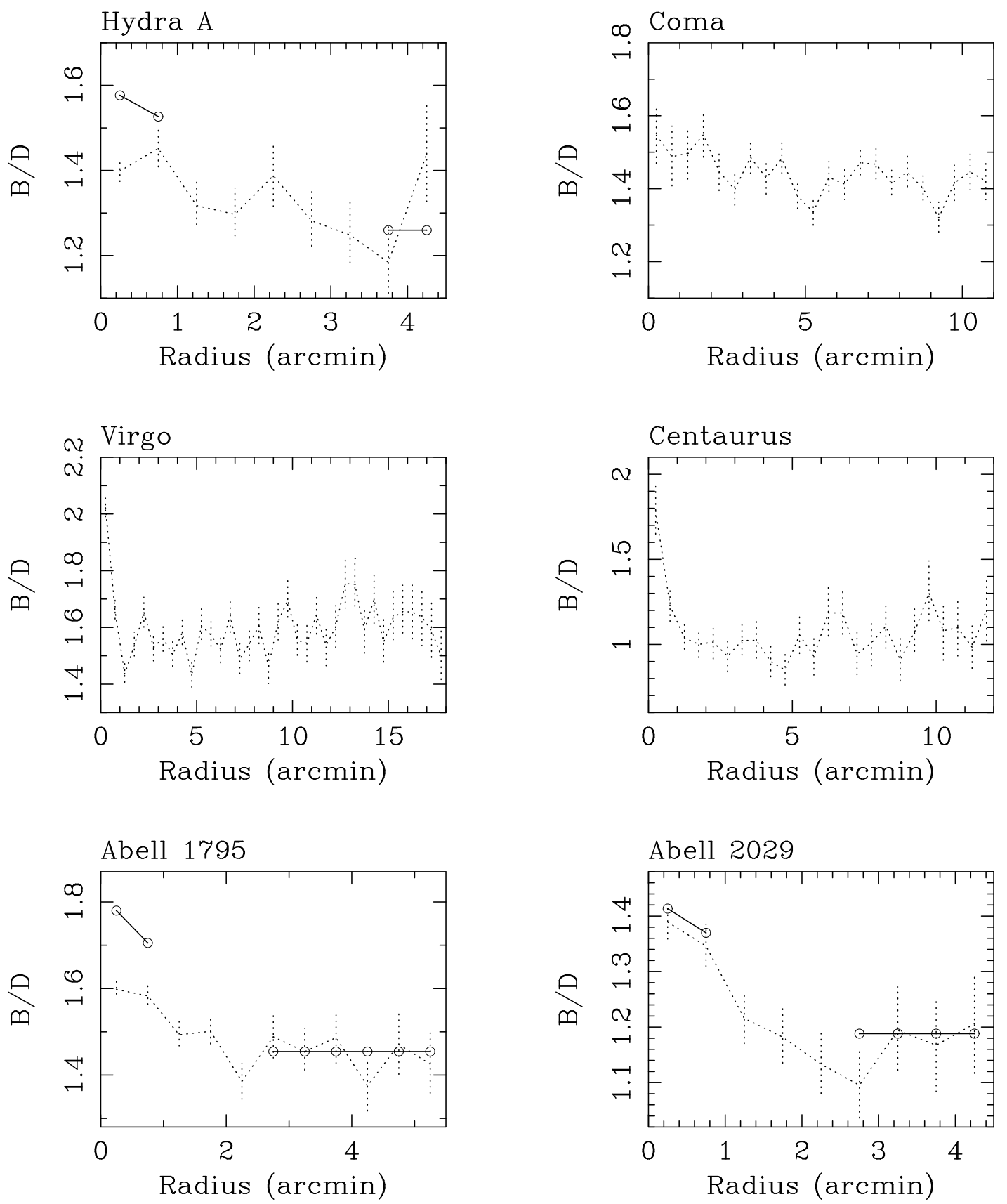

Figure 3. - continued

the central bin of the Centaurus data. We therefore defer detailed study of the data for the Virgo and Centaurus clusters to future work. (The relatively poor quality of the data for Abell 3112 and Abell 4059 also prohibit any detailed comparison with the deprojection code.) We note that hotter clusters do not generally exhibit metallicity gradients (e.g. Ohashi et al. 1996) and the effects of metallicity gradients on the X-ray colour profiles of such systems will, in any case, be small ( $c f$. Figs. 4,5).

The agreement between the observed and predicted $\mathrm{C} / \mathrm{D}$ ratios is remarkably good. For the best data sets (Abell
426, Abell 1795 and Abell 2199; see also Fig. 13) the agreement is especially good. In all other cases the predicted C/D values either agree with, or slightly under-predict, the observed values. Under-prediction in the central bin may again indicate a small drop in the ambient cluster temperature.

\subsection{The $\mathrm{B} / \mathrm{D}$ ratios and intrinsic absorption}

As with the $\mathrm{C} / \mathrm{D}$ results, the $\mathrm{B} / \mathrm{D}$ colour profiles shown in Fig. 3 are essentially flat at large radii $\left(r>r_{\text {cool }}\right)$. The observed and predicted values at large radii are generally in 
Table 5. $\mathrm{C} / \mathrm{D}$ and $\mathrm{B} / \mathrm{D}$ values at large radii

\begin{tabular}{cccccc} 
& & \multicolumn{2}{c}{ C/D } & \multicolumn{2}{c}{ B/D } \\
cluster & radii & observed & predicted & observed & predicted \\
\hline & & & & & \\
Abell 85 & $3.0-6.0$ & $2.58 \pm 0.08$ & 2.58 & $1.23 \pm 0.05$ & 1.20 \\
Abell 3112 & $3.0-6.0$ & $2.83 \pm 0.19$ & 2.80 & $1.34 \pm 0.11$ & 1.32 \\
Abell 426 & $6.0-15.0$ & $2.31 \pm 0.03$ & 2.24 & $0.82 \pm 0.01$ & 0.74 \\
Abell 478 & $2.5-4.5$ & $1.83 \pm 0.05$ & 2.07 & $0.39 \pm 0.02$ & 0.58 \\
Abell 496 & $3.6-9.0$ & $2.78 \pm 0.07$ & 2.64 & $1.18 \pm 0.04$ & 1.17 \\
Abell 644 & $3.0-7.2$ & $2.40 \pm 0.09$ & 2.35 & $0.89 \pm 0.04$ & 0.90 \\
Hydra A & $3.0-4.5$ & $2.72 \pm 0.10$ & 2.77 & $1.26 \pm 0.05$ & 1.20 \\
Coma & $2.0-12.0$ & $2.69 \pm 0.02$ & 2.61 & $1.42 \pm 0.01$ & 1.33 \\
Virgo & $10.0-12.0$ & $3.04 \pm 0.05$ & 3.07 & $1.57 \pm 0.04$ & 1.48 \\
Centaurus & $6.0-12.0$ & $2.66 \pm 0.09$ & 2.58 & $1.07 \pm 0.04$ & 0.97 \\
Abell 1795 & $2.5-5.5$ & $2.67 \pm 0.04$ & 2.76 & $1.46 \pm 0.03$ & 1.40 \\
Abell 2029 & $3.0-4.5$ & $2.46 \pm 0.09$ & 2.58 & $1.19 \pm 0.05$ & 1.19 \\
MKW3s & $3.0-4.2$ & $2.85 \pm 0.19$ & 2.99 & $1.33 \pm 0.11$ & 1.38 \\
Abell 2199 & $4.0-8.0$ & $2.93 \pm 0.04$ & 2.78 & $1.57 \pm 0.02$ & 1.43 \\
Cyg A & $1.5-3.0$ & $2.01 \pm 0.10$ & 1.91 & $0.44 \pm 0.04$ & 0.39 \\
Sersic 159 & $3.0-7.8$ & $3.15 \pm 0.17$ & 3.02 & $1.74 \pm 0.11$ & 1.48 \\
Abell 2597 & $1.8-3.0$ & $2.79 \pm 0.21$ & 2.83 & $1.34 \pm 0.16$ & 1.35 \\
Abell 4059 & $4.2-8.4$ & $2.79 \pm 0.17$ & 2.91 & $1.26 \pm 0.09$ & 1.44
\end{tabular}

Notes: The observed and predicted $\mathrm{C} / \mathrm{D}$ and $\mathrm{B} / \mathrm{D}$ ratios in the outer regions of the clusters. Column 2 lists the range of radii (in arcmin) that define the 'outer regions'. Columns 3 and 5 summarize the observed C/D and B/D values and $1 \sigma$ errors. Columns 4 and 6 list the predicted ratios for an isothermal plasma, at the temperature and redshift of the cluster. Galactic column densities (Table 3) and a metallicity of $0.5 Z_{\odot}$ are assumed. The general agreement between the observed and predicted $\mathrm{C} / \mathrm{D}$ and $\mathrm{B} / \mathrm{D}$ ratios demonstrates the validity of the isothermal assumption in these regions, and the absence of excess absorbing material at these radii (see discussion in Section 5.3).

Table 6. Absorption results from the B/D ratio

\begin{tabular}{ccccc} 
Cluster & $N_{\mathrm{H}}$ & $\Delta N_{\mathrm{H}}^{\prime}$ & $\Delta N_{\mathrm{H}}(1)$ & $\Delta N_{\mathrm{H}}(2)$ \\
\hline & & & & \\
Abell 85 & 2.96 & $2.34 \pm 0.60$ & $5.4_{-1.6}^{+1.7}$ & $10.5 \pm 2.7$ \\
Abell 426* & 13.7 & $0.50 \pm 0.55$ & $1.0_{-1.0}^{+1.2}$ & $2.3 \pm 2.5$ \\
Abell 478 & 20.0 & $7.4 \pm 0.63$ & $20.1_{--2}^{+2.7}$ & $33.3 \pm 2.8$ \\
Abell 496 & 4.27 & $2.57 \pm 0.62$ & $6.0_{-1.6}^{+1.9}$ & $11.6 \pm 2.8$ \\
Hydra A & 4.84 & $3.07 \pm 0.41$ & $7.5_{-1.2}^{+1.4}$ & $13.8 \pm 1.8$ \\
Abell 1795 & 1.11 & $2.20 \pm 0.20$ & $5.0_{-0.5}^{+0.6}$ & $9.9 \pm 0.9$ \\
Abell 2029 & 3.05 & $0.83 \pm 0.42$ & $1.7_{-0.9}^{+1.0}$ & $3.7 \pm 1.9$ \\
MKW3s & 2.86 & $7.29 \pm 0.88$ & $11.7_{-1.7}^{+1.9}$ & $32.8 \pm 4.0$ \\
Abell 2199 & 0.87 & $2.67 \pm 0.25$ & $6.3_{-0.7}^{+0.8}$ & $12.0 \pm 1.1$ \\
Cygnus A & 32.8 & $5.5 \pm 1.0$ & $13.4_{--.9}^{+3.3}$ & $24.8 \pm 4.5$ \\
Sersic 159 & 1.80 & $1.87 \pm 0.48$ & $4.2_{-1.2}^{+1.2}$ & $8.4 \pm 2.2$ \\
Abell 2597 & 2.46 & $2.36 \pm 0.58$ & $5.5_{-1.6}^{+1.6}$ & $10.6 \pm 2.6$
\end{tabular}

The intrinsic column densities and $1 \sigma$ errors (in units of $10^{20}$ atom $\mathrm{cm}^{-2}$ ) for the central 30 arcsec regions of the clusters, inferred from the B/D analysis (Section 5.3). Column 2 lists the Galactic column densities. Column $3\left(\Delta N_{\mathrm{H}}^{\prime}\right)$ summarizes the excess column densities determined with the simple uniform absorbing screen model. Column $4\left[\Delta N_{\mathrm{H}}(1)\right]$ lists the corrected intrinsic column densities, determined from equation 4 , with the partial covering model (the preferred model). A covering fraction, $f=0.5$, and a photon energy, $E=0.4 \mathrm{keV}$, have been assumed for all clusters except Abell 478 and Cygnus A (which have much higher Galactic column densities) for which $E=0.5$ $\mathrm{keV}$ is adopted. For MKW3s the observed $\Delta N_{\mathrm{H}}^{\prime}$ is inconsistent with $f=0.5$ and $f=0.75$ is used instead. Column $5\left[\Delta N_{\mathrm{H}}(2)\right]$ lists the corrected intrinsic column densities determined with equation 5, for the multilayer absorption model. An intermediate correction factor of 4.5 has been assumed. ${ }^{*}$ Between radii of $1-2 \operatorname{arcmin}$ in Abell 426, the corrected intrinsic column density is $\Delta N_{\mathrm{H}}(1) \sim 5-10 \times 10^{20}$ atom $\mathrm{cm}^{-2}$. 

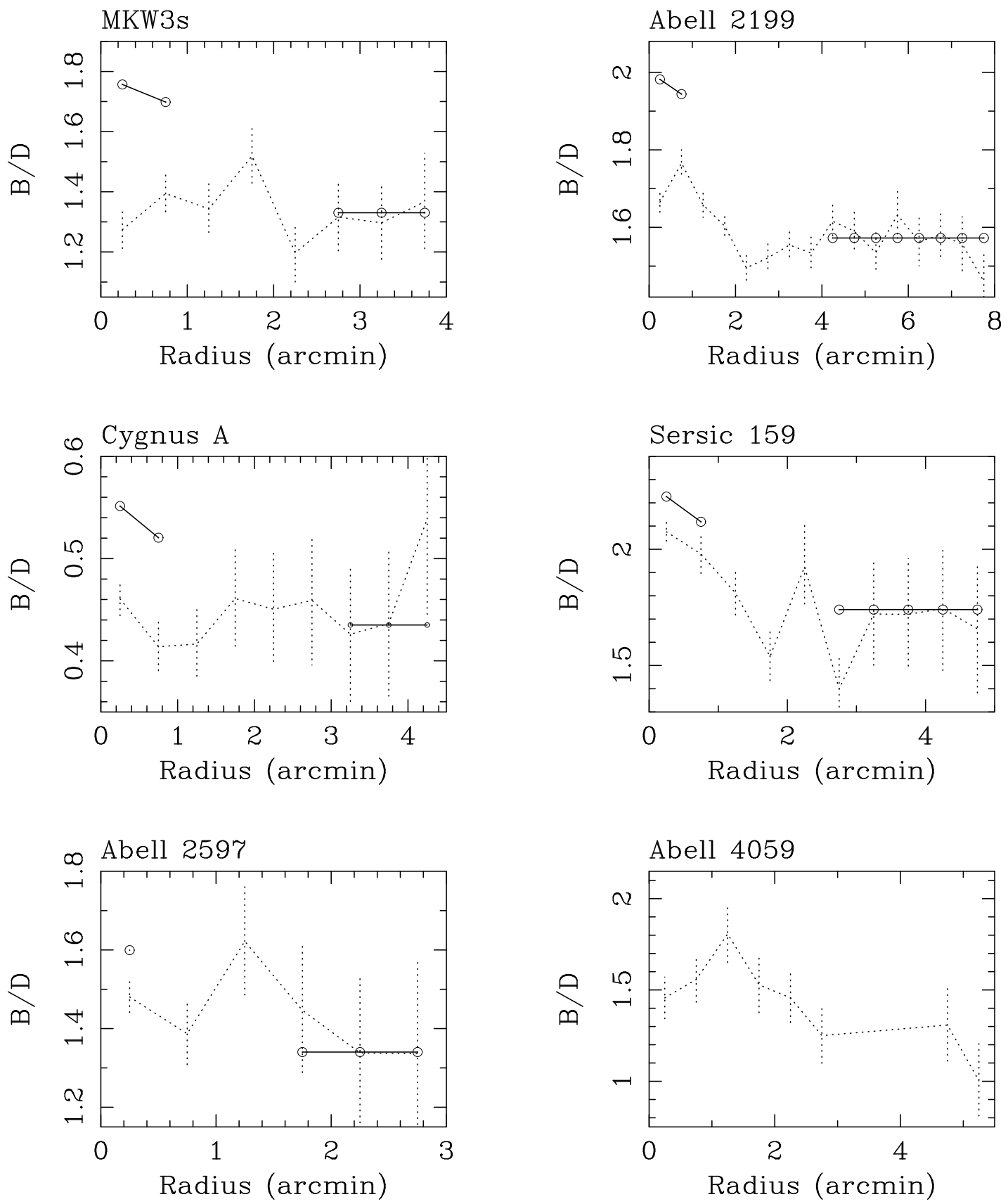

Figure 3. - continued

good agreement (although with a few exceptions detailed below) indicating that the emission from these regions can be well-described by a simple isothermal model, with the temperatures and Galactic column densities listed in Table 3. At small radii, many of the $\mathrm{B} / \mathrm{D}$ profiles increase. However, the gradients are not as steep as for the $\mathrm{C} / \mathrm{D}$ results, and for a number of the clusters the B/D profiles are essentially flat or even decrease slightly in the innermost bins (e.g. Abell 478).

The most notable exception to the agreement between the observed and predicted colour ratios at large radii is
Abell 478, for which the B/D (and C/D) ratio implies a Galactic column density of $\sim 3 \times 10^{21}$ atom $\mathrm{cm}^{-2}$, an excess of $\sim 10^{21}$ atom $\mathrm{cm}^{-2}$ over the value listed in Table 3 . In contrast, the data for Abell 426 require a column density of $\sim 1.1 \times 10^{21}$ atom $\mathrm{cm}^{-2}$, slightly less than the Galactic value. For the Coma cluster, the $\mathrm{B} / \mathrm{D}$ and $\mathrm{C} / \mathrm{D}$ ratios imply an emission-weighted temperature at large radii of $\sim 6 \mathrm{keV}$, somewhat less than the $8 \mathrm{keV}$ listed in Table 3. For Abell 2199 the data suggest an emission weighted temperature of $\sim 3.5 \mathrm{keV}$ and a column density of $\sim 5 \times 10^{19}$ atom $\mathrm{cm}^{-2}$. Similarly, the data for the Virgo cluster imply a temperature 

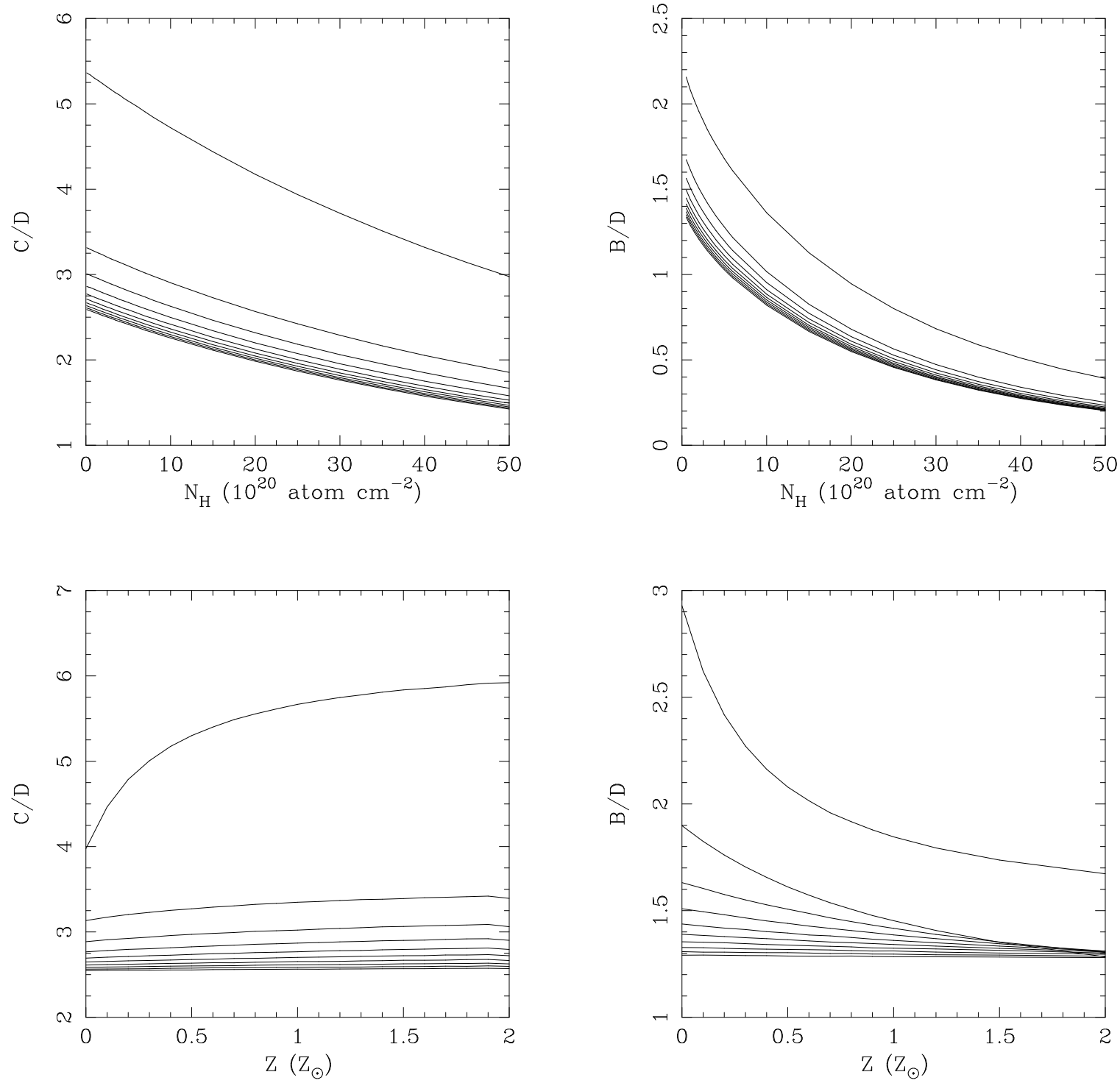

Figure 4. Theoretical predictions for the $\mathrm{C} / \mathrm{D}$ and $\mathrm{B} / \mathrm{D}$ ratios for isothermal gas at temperatures of $1-10 \mathrm{keV}$. (a) C/D as a function of column density $\left(Z=0.5 Z_{\odot}\right.$ fixed). (b) B/D as a function of column density $\left(Z=0.5 Z_{\odot}\right.$ fixed). (c) $\mathrm{C} / \mathrm{D}$ as a function of metallicity $\left(N_{\mathrm{H}}=10^{20}\right.$ atom $\mathrm{cm}^{-2}$ fixed). (d) B/D as a function of metallicity $\left(N_{\mathrm{H}}=10^{20}\right.$ atom $\mathrm{cm}^{-2}$ fixed). In all cases the temperature of the gas increases with decreasing $\mathrm{C} / \mathrm{D}$ and $\mathrm{B} / \mathrm{D}$ along the $\mathrm{y}$ axis.

of $\sim 2 \mathrm{keV}$ and a column density of $\sim 1.3 \times 10^{20}$ atom $\mathrm{cm}^{-2}$, both slightly less than the nominal values.

Following our success in applying the deprojection code to the prediction of the C/D profiles (Section 5.2), we have used the same technique to predict the $\mathrm{B} / \mathrm{D}$ profiles. The predicted data are shown as the circled points in Fig. 3 . The results demonstrate a clear tendency for the predicted values to overestimate the observations in the central regions.

The theoretical curves presented in Figs. 4 and 5 show that the most plausible interpretation for the central deficits in the $\mathrm{B} / \mathrm{D}$ observations, with respect to the predicted data, is the presence of intrinsic absorbing material in the clusters. The excess column densities required to bring the observed and predicted $\mathrm{B} / \mathrm{D}$ values for the central bins into agreement are summarized in Table 6. These values have been calcu- lated using XSPEC models for the projected spectrum of the central 30 arcsec (radius) in each cluster, as implied by the deprojection analysis. The excess absorption has then been modelled as a uniform screen in front of the cluster. Note that since the observed $\mathrm{C} / \mathrm{D}$ ratios for the central bins sometimes (slightly) exceed the predicted values, the column densities listed in Table 6 may (slightly) underestimate the true values.

Where comparisons between the observed and predicted $\mathrm{B} / \mathrm{D}$ ratios are possible for more than just the central bin, the data suggest that the column density increases with decreasing radius within the cooling flow. This is consistent with a picture wherein the intrinsic absorber is accumulated from material deposited by the cooling flow. The only exception is Abell 426 (the Perseus Cluster) for which the ap- 

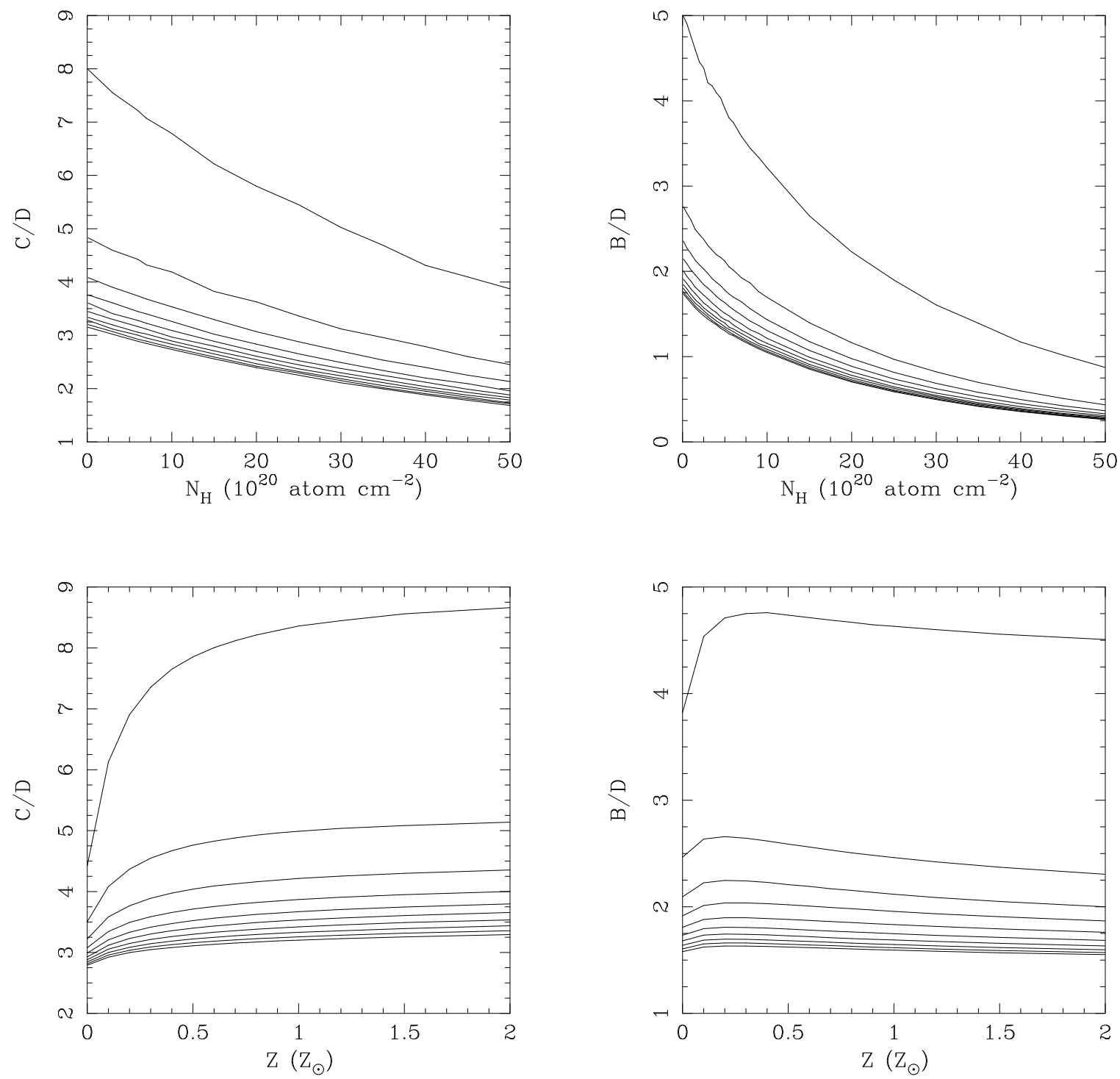

Figure 5. Theoretical predictions for the $\mathrm{C} / \mathrm{D}$ and $\mathrm{B} / \mathrm{D}$ ratios for gas cooling at constant pressure from and upper temperature of 1-10 $\mathrm{keV}$ to a lower temperature of $.001 \mathrm{keV}$. (a) $\mathrm{C} / \mathrm{D}$ as a function of column density $\left(Z=0.5 Z_{\odot}\right.$ fixed). (b) B/D as a function of column density $\left(Z=0.5 Z_{\odot}\right.$ fixed). (c) $\mathrm{C} / \mathrm{D}$ as a function of metallicity $\left(N_{\mathrm{H}}=10^{20}\right.$ atom $\mathrm{cm}^{-2}$ fixed). (d) B/D as a function of metallicity $\left(N_{\mathrm{H}}=10^{20}\right.$ atom $\mathrm{cm}^{-2}$ fixed). In all cases the temperature of the gas increases with decreasing $\mathrm{C} / \mathrm{D}$ and $\mathrm{B} / \mathrm{D}$ along the $\mathrm{y}$ axis.

parent excess column density at radii of $1-2$ arcmin is $5-10$ times larger than for the central bin. It is likely that the results for the central regions of Abell 426 are affected by the complex morphology of the cluster (Böhringer et al. 1993) and emission from the active nucleus of the central galaxy. Note also that although the presence of temperature and metallicity gradients complicate the interpretation of the $\mathrm{B} / \mathrm{D}$ data for the Virgo and Centaurus clusters, the comparison of their $\mathrm{B} / \mathrm{D}$ ratios, which are only observed to rise within the central arcminute or so, with their $\mathrm{C} / \mathrm{D}$ ratios, which in contrast rise steadily within radii of 7 and 3 arcmin respectively, again indicates the presence of distributed absorbing material.

Our results confirm that excess absorption is common in cooling flows. All of the cooling flow clusters in our sam- ple, for which an X-ray colour profile/deprojection comparison is possible, show evidence for intrinsic absorption. Note also that the cooling flow with the lowest intrinsic column density, Abell 2029, is also the only cluster with a Central Cluster Galaxy (CCG) that does not exhibit optical emission lines (e.g. Hu, Cowie \& Wang 1985; Johnstone, Fabian $\&$ Nulsen 1987). This suggests a link between the presence of X-ray absorbing gas in cooling flows and the production of optical emission lines in CCGs. Such a result is compatible with models in which the emission lines are powered by massive stars that collapse out of the cooled gas accumulated by the cooling flows (Allen 1995). Processes such as turbulent mixing layers and shocks in the X-ray absorbing medium may also contribute significantly to the observed emission-line flux (Crawford \& Fabian 1992). 

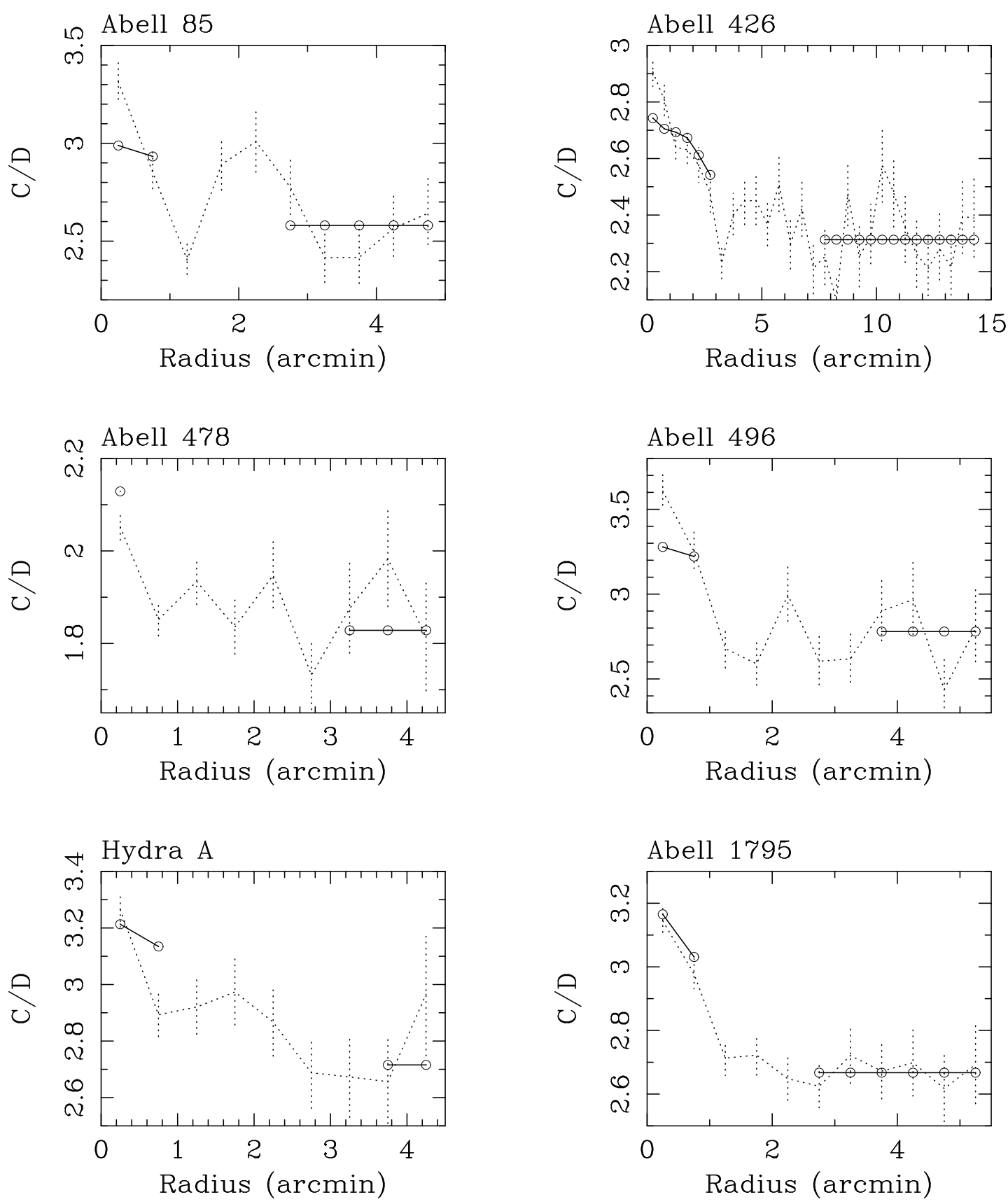

Figure 6. A comparison of the observed C/D profiles (as shown in Fig. 2) with the ratios predicted from the deprojection code (Section $5.2)$.

Finally, we note that although the analysis of the B/D profiles apparently suggests intrinsic column densities of only a few $10^{20}$ atom $\mathrm{cm}^{-2}$ through the centres of cooling flows, when modelling the absorber as a uniform screen in front of the clusters, the true intrinsic column densities are likely to be $2-5$ times higher, once systematic effects associated due to the spatial distribution of the absorbing gas are accounted for (see Section 6).

\subsection{The absorption signature in the ' $A$ ' band}

The $\mathrm{B} / \mathrm{D}$ ratio provides the most reliable probe of the intrinsic column density in the clusters. As noted in Section 1, at energies below $0.40 \mathrm{keV}$ (the lower limit of the B band) the point spread function of the PSPC broadens significantly. In the $A_{1}(0.1-0.2 \mathrm{keV})$ band a significant fraction of the flux is scattered (somewhat asymmetrically) to radii $>1$ arcmin. In the $A_{2}$ band, the PSF is better behaved, but the FWHM is still $\sim 1.7$ times broader than in bands B-D. [Note that the $\mathrm{B} / \mathrm{D}$ ratio also has the advantage of being applicable 

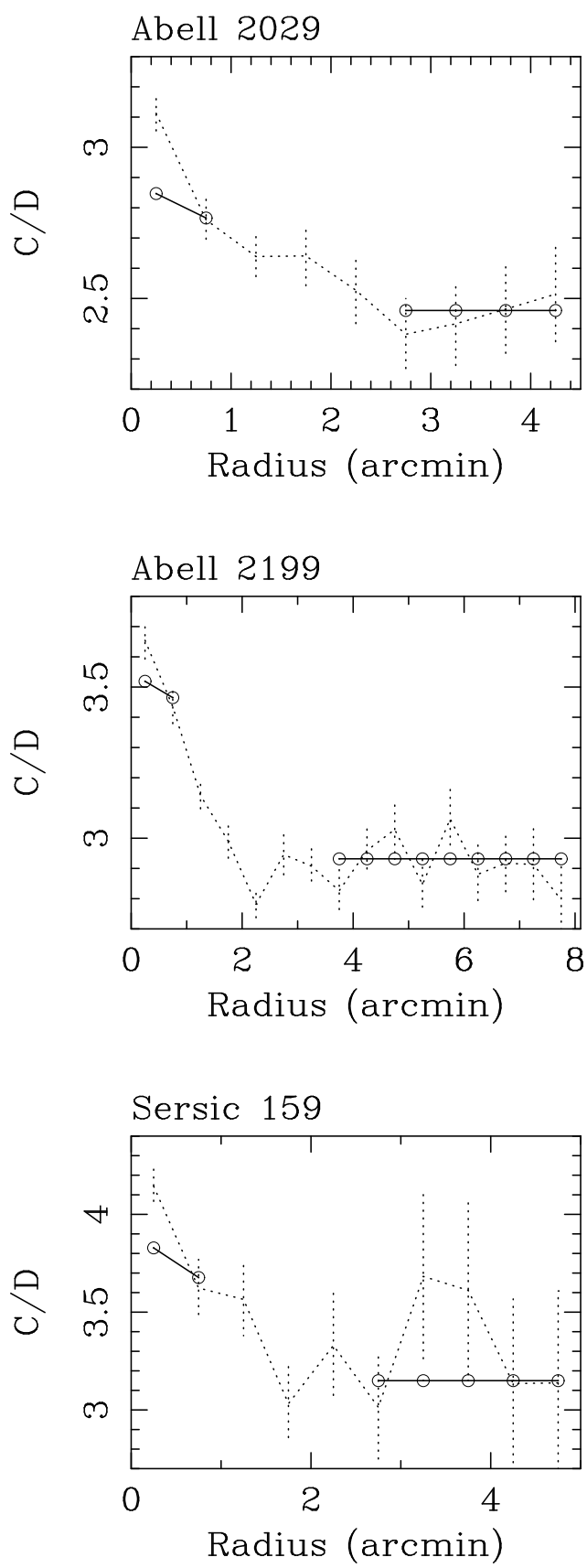
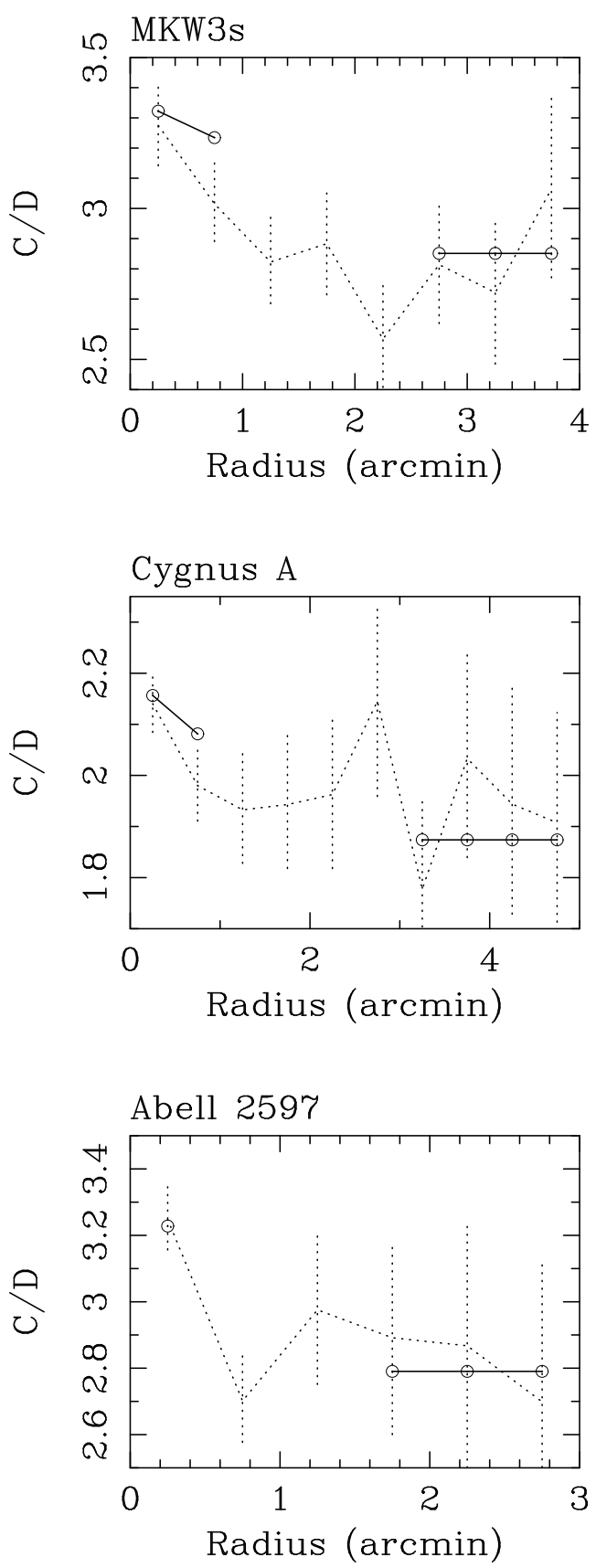

Figure 6. - continued

to clusters with a wide range of Galactic column densities, whereas the A band data are lost for clusters behind Galactic column densities $\gtrsim 10^{21}$ atom $\mathrm{cm}^{-2}$.]

We have carried out a search for the signature of intrinsic absorption in the $A_{2}$ data, by forming profiles in the ratio $A_{2} / \mathrm{D}^{\prime}$, where $\mathrm{D}^{\prime}$ is the $\mathrm{D}$ band profile convolved with a Gaussian filter of 30 arcsec FWHM. For the two best data sets for which this test is applicable, Abell 1795 and Abell 2199 , we determine excess column densities in the central bin of $\sim 1-2 \times 10^{19}$ atom $\mathrm{cm}^{-2}$. These column densities are significantly less than those inferred from the B/D anal- ysis. However such results are to be expected if the absorber is spatially distributed throughout the cooling flows, as discussed in Section 6, below. Note also that the presence of an absorption signature in the $A_{2}$ data implies that absorbers other than Oxygen (which has its $\mathrm{K}$ edge at $0.53 \mathrm{keV}$ in low temperature gas) must be present in the clusters.

\subsection{The ages of the cooling flows}

Throughout this Section we have limited our comparison of the observed and predicted X-ray colour profiles to radii 

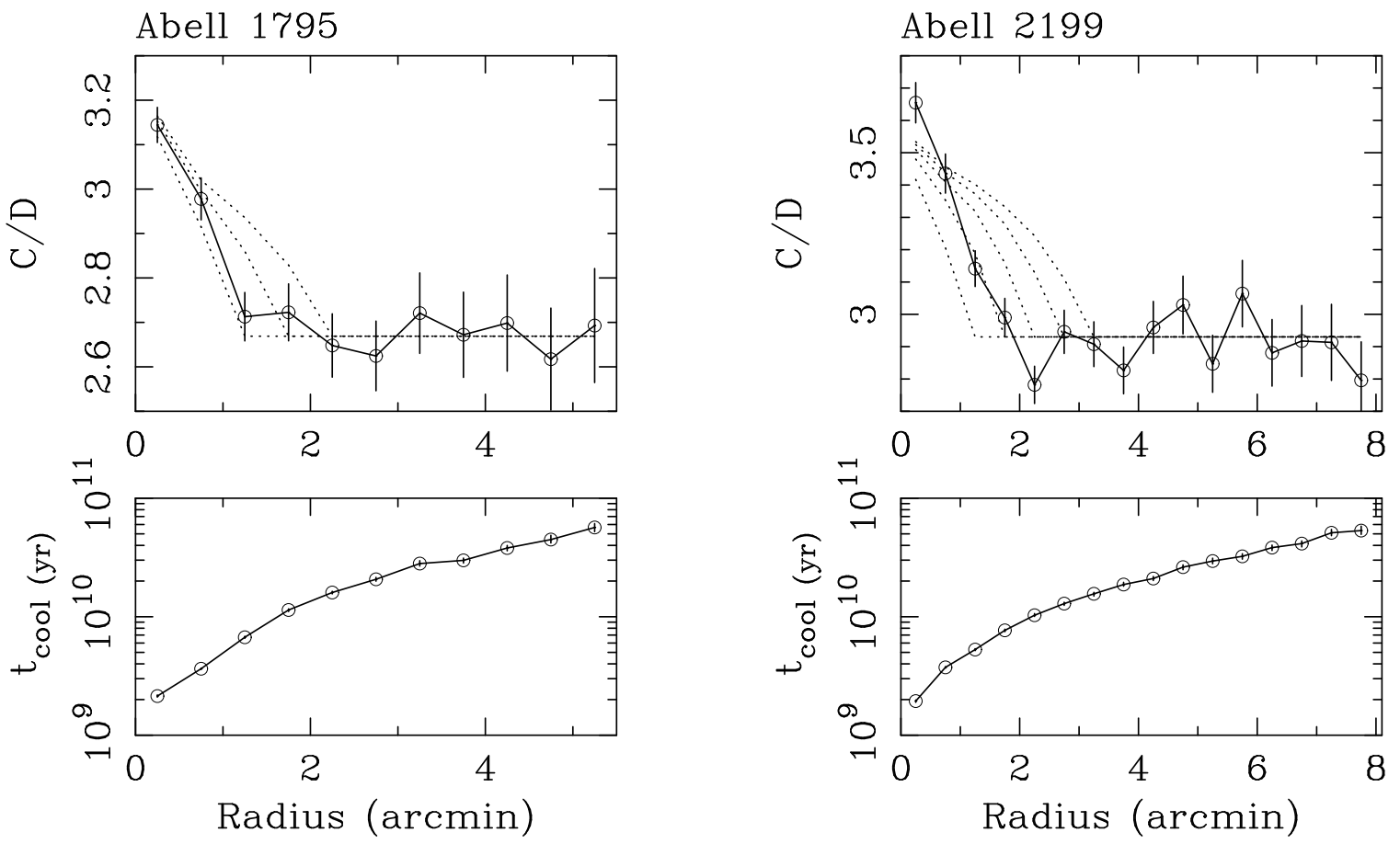

Figure 7. (Upper panels) Comparison of the observed C/D colour profiles with the predictions (dotted curves) from the deprojection code, as a function of the age of the cooling flows. For Abell 1795, the predictions are shown for cooling cut-off times of 6.7,11.4 and $16.0 \times 10^{9} \mathrm{yr}$, corresponding to ages of $3.6-6.7 \times 10^{9}, 6.7-11.4 \times 10^{9}$, and $11.4-16.0 \times 10^{9} \mathrm{yr}$, respectively. For Abell 2199 the cooling cut-off points are 5.3,7.7,10.3,12.9 and $15.6 \times 10^{9} \mathrm{yr}$, implying ages of $3.7-5.3,5.3-7.7,7.7-10.3,10.3-12.9$ and $12.9-15.6 \times 10^{9}$ yr. (Lower panels) The mean cooling time as a function of radius in the clusters.

where the mean cooling time of the cluster gas is $\leq 5 \times 10^{9}$ yr. However, for the best data sets, the data from larger radii allow constraints to be placed on the age of the cooling flows (where the age is presumably related to the time since the central regions of the clusters were last disrupted by a major subcluster merger event). For these data sets, the deprojection code has been used to predict $\mathrm{C} / \mathrm{D}$ profiles throughout the clusters, as a function of cooling-flow age. At radii where the mean cooling time of the gas is equal to or exceeds a specified value, the cluster is assumed to be isothermal with no mass deposition occurring. At interior radii, where the mean cooling time is less than this value, the predictions are calculated in the manner described in Section 5.2

Fig. 7 shows the observed C/D profiles and the predicted results, as a function of cooling flow age, for the two best data sets in the sample, Abell 1795 and Abell 2199 . Also plotted are the mean cooling times as a function of radius in the clusters. For Abell 1795, the data are best described by an age of between 3.6 and $6.7 \times 10^{9} \mathrm{yr}\left(6.7 \times 10^{9}\right.$ yr being the mean cooling time at the radius where the $\mathrm{C} / \mathrm{D}$ ratio rises, and $3.6 \times 10^{9} \mathrm{yr}$ the mean cooling time for the next interior bin.) For Abell 2199, the data suggest an age of $5.3-7.7 \times 10^{9}$ yr. The results also imply mass deposition rates in the clusters somewhat less than the nominal values quoted in Table 3 (which are calculated for an assumed age of $1.3 \times 10^{10} \mathrm{yr}$.) For Abell 1795 we determine a revised mass deposition rate of $\sim 250 \mathrm{M}_{\odot} \mathrm{yr}^{-1}$. For Abell 2199 the revised mass deposition rate is $\sim 80 \mathrm{M}_{\odot} \mathrm{yr}^{-1}$. These results are approximately a factor 2 less than the values of $\sim 450$ and $160 \mathrm{M}_{\odot} \mathrm{yr}^{-1}$ determined under the standard deprojection assumptions, but are in good agreement with the rates inferred from the analysis of Einstein SSS spectra for these clusters by White et al. (1991).

In general, the data for the other clusters do not place firm constraints on the age of the cooling flows. However, for Abell 496 the data suggest an age of between 3.3 and $6.8 \times$ $10^{9} \mathrm{yr}$, and a mass deposition rate, $\dot{M} \sim 50 \mathrm{M}_{\odot} \mathrm{yr}^{-1}$ (again in good agreement with the SSS spectral results). For Sersic 159 , the age and mass deposition rate are $6.4-10.5 \times 10^{9}$ yr and $\dot{M} \sim 220 \mathrm{M}_{\odot} \mathrm{yr}^{-1}$, respectively. For Abell 2597, the values are $2.5-5.1 \times 10^{9} \mathrm{yr}$ and $\dot{M} \sim 200 \mathrm{M}_{\odot} \mathrm{yr}^{-1}$. In contrast the data for both Hydra A and Abell 2029 are consistent with the cooling flows having been undisturbed for close to a Hubble time.

In detail, the relationship between the age of the cooling flow and the mean cooling time in the region where the $\mathrm{C} / \mathrm{D}$ ratio changes will be more complex than discussed above, and will depend on (undetermined) factors relating to the distribution of density inhomogeneities in the cluster gas. However, the uncertainties associated with such effects should not much exceed those quoted above.

These results illustrate how standard image deprojection analyses, which assume that cooling flows have existed 


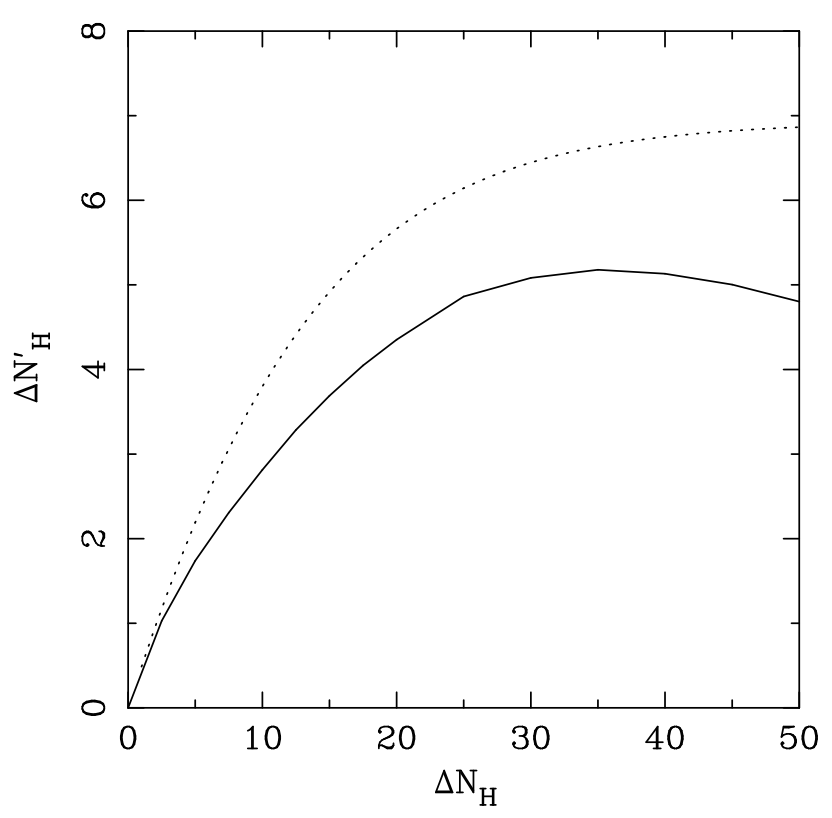

Figure 10. The relationship between the apparent excess column density, $\Delta N_{\mathrm{H}}^{\prime}$, and the true intrinsic column density, $\Delta N_{\mathrm{H}}$, determined from the analytical expression in equation 4 (dotted curve) and spectral simulations (solid curve). For the spectral simulations a Galactic column density of $10^{20}$ atom $\mathrm{cm}^{-2}$ has been assumed. Both axes are in units of $10^{20}$ atom $\mathrm{cm}^{-2}$.

for a Hubble time, can overestimate the integrated mass deposition rates in clusters by up to a factor of 2. Future studies, with the improved spectral and spatial resolution of AXAF, will allow such properties to be explored in greater detail. The aging of cooling flows may provide a powerful probe of the evolutionary history of clusters.

\section{THE INTERPRETATION OF FITTED COLUMN DENSITIES}

In this Section we further examine the results on intrinsic absorption in the cooling flows. We discuss how measurements of intrinsic column densities, determined from fits with uniform foreground screen absorption models, will naturally underestimate the true intrinsic column densities when the absorbing gas is spatially distributed throughout the cooling flows. In particular, we explore two limiting distributions for the absorbing gas; partial covering (which will apply if the absorbing gas is clumped on large scales) and a homogeneous distribution of absorbing material throughout the $\mathrm{X}$-ray emitting region, which we refer to as the 'multilayer' model. We compare the absorption measurements from the ROSAT X-ray colour profile analysis to previous results from the Einstein SSS (White et al. 1991) and ASCA. Finally in this Section, we comment on the mass of absorbing gas in the clusters and on the role of this material as a sink for the cooling gas.

\subsection{The effects of partial covering}

The use of simple uniform-screen absorption models in the analysis of cluster spectra can lead to significant underestimates of intrinsic column densities. A simple illustration of this, in the case where the absorbing material covers only a fraction, $f$, of the intrinsic emission, is given in Figs. 8(a) and (c). The figures show the column densities inferred from fits with simple uniform (foreground) screen absorption models to simulated cluster spectra incorporating intrinsic absorption with partial covering. For the simulations we assume a cluster temperature of $5 \mathrm{keV}$, a metallicity of 0.5 solar, and a redshift, $z=0.01$. The cluster is assumed to lie behind a Galactic column density, $N_{\mathrm{H}}=10^{20}$ atom $\mathrm{cm}^{-2}$, and to be intrinsically absorbed by an excess column density, $\Delta N_{\mathrm{H}}$, which covers a fraction, $f$, of the emitted flux. Intrinsic column densities of $\Delta N_{\mathrm{H}}=10^{21}$ and $\Delta N_{\mathrm{H}}=5 \times 10^{21}$ atom $\mathrm{cm}^{-2}$, and covering fractions of $0.1-1.0$, were examined. [These column densities span the range of values inferred from Einstein Observatory SSS and ASCA observations of cooling flows; White et al. (1991), Fabian et al. (1994), Allen et al. (1996a), Allen et al. (1996b), Fabian et al. (1996).]

The simple partial covering distribution provides a crude approximation to the likely situation in an intrinsically absorbed cooling flow. In the simplest case of a geometrically thin absorbing screen of uniform column density, the observed emission can be modelled by two components, accounting for the emission from in front of and behind the absorber, respectively. If the absorber spans the entire region of interest, the components should have equal intrinsic fluxes, and be absorbed by column densities $N_{\mathrm{H}}$ and $N_{\mathrm{H}}+\Delta N_{\mathrm{H}}$. In this case, the covering fraction of the intrinsic absorber is 0.5 . If the intrinsic absorber is present over only part of the region of interest, as would be the case if the extent of the absorber were less than the region being analysed, the covering fraction will be less than 0.5. (With a real cooling flow, variations in both column density and covering fraction are likely.)

The fits to the simulated spectra were carried out in both the $0.1-2.0 \mathrm{keV}$ and $0.4-2.0 \mathrm{keV}$ energy bands. The temperature and metallicity were fixed at $5 \mathrm{keV}$ and 0.5 $\mathrm{Z}_{\odot}$, respectively. For covering fractions less than unity, the column densities determined from fits with uniform-screen $(f=1)$ absorption models significantly underestimate the 'true' absorption. For an intrinsic column density, $\Delta N_{\mathrm{H}}=$ $5 \times 10^{21}$ atom $\mathrm{cm}^{-2}$, and a covering fraction, $f=0.5$, a fit to the $0.4-2.0 \mathrm{keV}$ spectrum implies a column density of $5.7 \times$ $10^{20}$ atom $\mathrm{cm}^{-2}$, an excess of only $4.7 \times 10^{20}$ atom $\mathrm{cm}^{-2}$ over the Galactic value. Thus the column density inferred from the spectral fit to the $0.4-2.0 \mathrm{keV}$ band data underestimates the true intrinsic column density by a factor of $\sim 10$. Even more dramatically, when the full $0.1-2.0 \mathrm{keV}$ energy range is used, the spectral fit implies an excess column density of only $4.5 \times 10^{19}$ atom $\mathrm{cm}^{-2}$, underestimating the true intrinsic column density by a factor of $\sim 100$ ( $c f$. Section 5.4). In a $\chi^{2}$ sense, however, the uniform absorption model still provides a statistically adequate description of the simulated spectra (for signal-to-noise ratios in the simulated spectra typical of PSPC cluster observations). Similar results are obtained for the simulations with $\Delta N_{\mathrm{H}}=10^{21}$ atom $\mathrm{cm}^{-2}$, although the underestimation of intrinsic column densities from the spectral fits is less severe. A fit to the $0.4-2.0 \mathrm{keV}$ spectrum, 

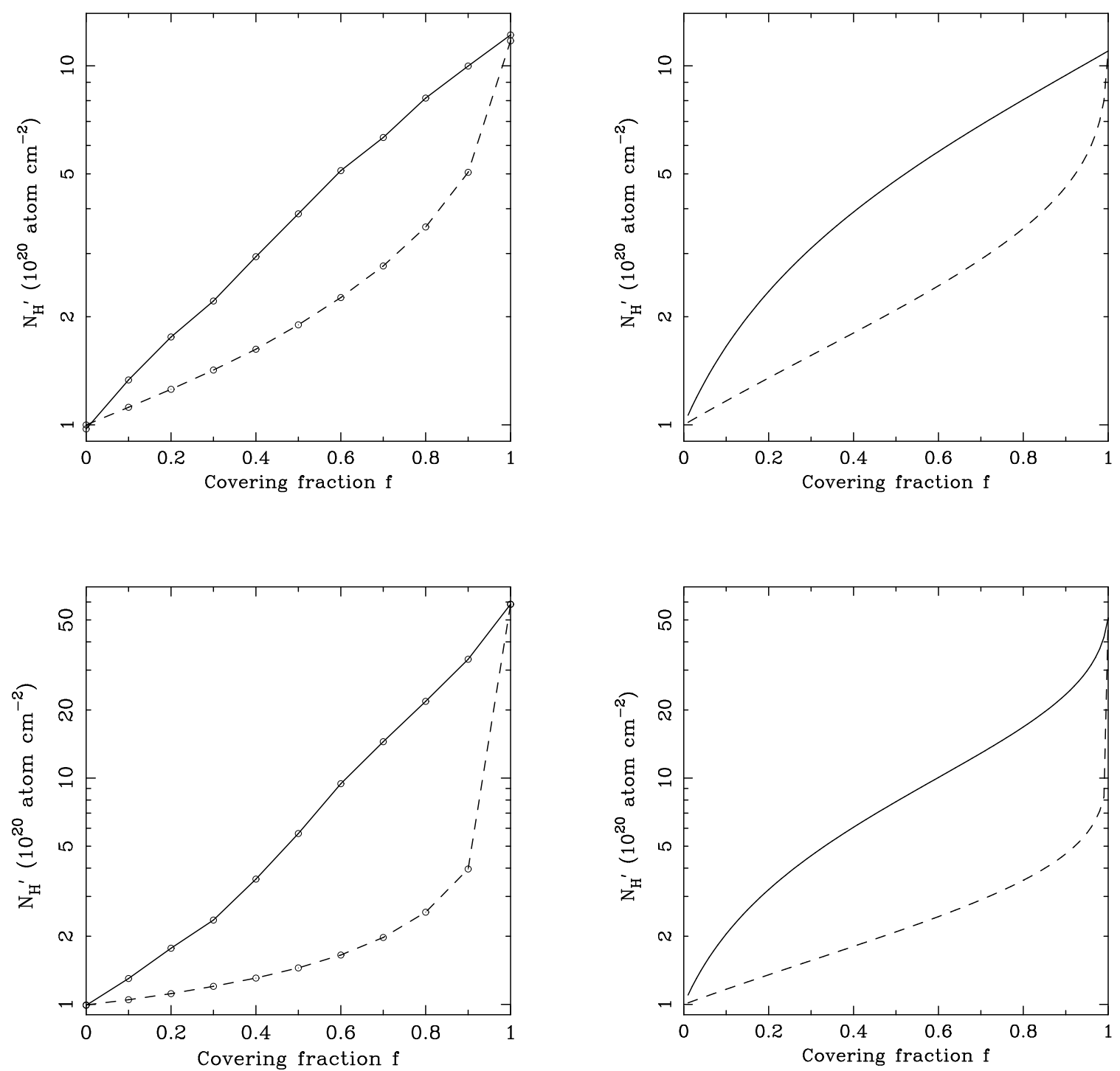

Figure 8. (a) The apparent column density, $N_{\mathrm{H}}^{\prime}$, as a function of the covering fraction, $f$, of the intrinsic absorber, determined from fits to the simulated spectra with simple uniform-foreground-screen absorption models (Section 6.1). A Galactic column density, $N_{\mathrm{H}}=10^{20}$ atom $\mathrm{cm}^{-2}$, and an intrinsic column density, $\Delta N_{\mathrm{H}}=10^{21}$ atom $\mathrm{cm}^{-2}$, are assumed. We adopt a fixed temperature of $5 \mathrm{keV}$, a metallicity $0.5 \mathrm{Z}_{\odot}$ and a redshift $z=0.01$. Results are shown for fits to both the full $0.1-2.0 \mathrm{keV}$ band (lower dashed curve) and the $0.4-2.0 \mathrm{keV}$ band (upper solid curve). (b) The similar results obtained from the analytical expression in equation 3 for $\Delta N_{\mathrm{H}}=10^{21}$ atom $\mathrm{cm}^{-2}$ and photon energies of $0.4 \mathrm{keV}$ (upper solid line) and $0.2 \mathrm{keV}$ (lower dashed line). (c) As for (a) but with $\Delta N_{\mathrm{H}}=5 \times 10^{21}$ atom $\mathrm{cm}^{-2}$. (d) As for (b) with $\Delta N_{\mathrm{H}}=5 \times 10^{21}$ atom $\mathrm{cm}^{-2}$.

with $f=0.5$, gives a measured column density of $3.9 \times 10^{20}$ atom $\mathrm{cm}^{-2}$, implying an excess column density a factor of $\sim 3$ less than the true value.

Such results are not sensitive to the precise emission spectrum assumed. If a cooling flow, rather than isothermal, model is used both for generating and fitting the simulated spectra, results very similar results to those plotted in Fig. 8 are obtained. Significant discrepancies only arise when different emission models are used for generating and fitting the simulated data.

In Fig. 9 we show the results from a consistency check in which compare the results from a simple, uniform-screen fit to a simulated cluster spectrum with $N_{\mathrm{H}}=10^{20}$ atom $\mathrm{cm}^{-2}, \Delta N_{\mathrm{H}}=10^{21}$ atom $\mathrm{cm}^{-2}$ and $f=0.5$, with the results from a fit to the observed spectrum for the central 2 arcmin (radius) region of Abell 1795. (The signal-to-noise ratio in the simulated spectrum has been adjusted to approximately match that in the Abell 1795 data.) In both cases, the fit to the $0.1-2.0 \mathrm{keV}$ spectrum implies an excess column density of $\sim 2 \times 10^{19}$ (whereas the B/D results for Abell 1795 imply a much larger intrinsic column density of $\sim 5 \times 10^{20}$ atom $\left.\mathrm{cm}^{-2}\right)$.

Some intuitive understanding of these results may be 

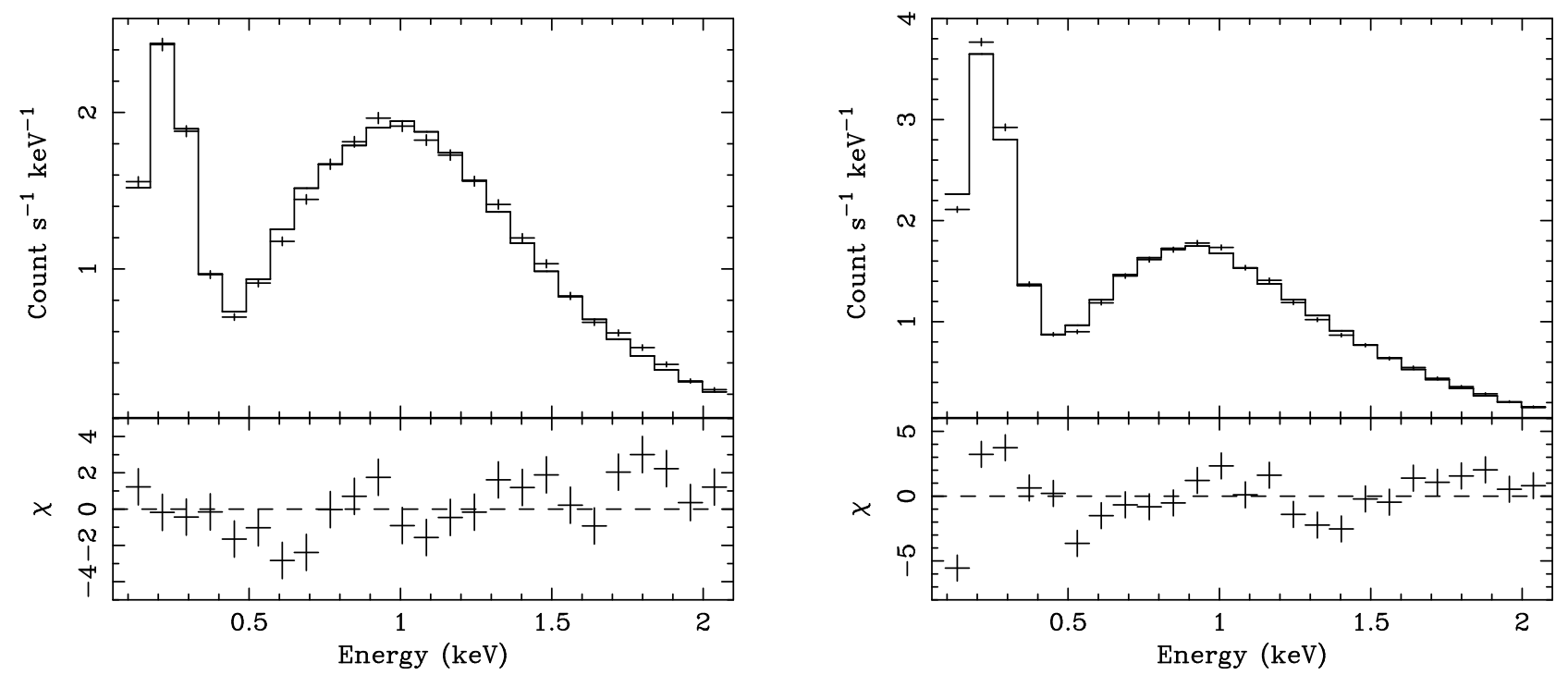

Figure 9. (a) Simulated spectrum of a $5 \mathrm{keV}$ cluster at a redshift $z=0.01$, absorbed by a Galactic column density of $10^{20}$ atom $\mathrm{cm}^{-2}$ (covering fraction unity) and an intrinsic column density of $10^{21}$ atom $\mathrm{cm}^{-2}$ (covering fraction $f=0.5$ ). The data are shown for the full $0.1-2.0 \mathrm{keV}$ range, with the best-fitting single temperature, uniform absorbing screen model overlaid. The best-fit column density is $1.25_{-0.11}^{+0.10} \times 10^{20}$ atom $\mathrm{cm}^{-2}$, implying an excess column density of $2.5_{-1.1}^{+1.0} \times 10^{19}$ atom $\mathrm{cm}^{-2}$. The lower panel shows the residuals to the fit. (b) The spectrum for the central 2 arcmin of Abell 1795, at a redshift $z=0.06334$, with the best-fitting single temperature, uniform absorbing screen model overlaid. The best fit column density is $1.27_{-0.04}^{+0.04} \times 10^{20}$ atom $\mathrm{cm}^{-2}$, implying an excess column density of $1.6_{-0.4}^{+0.4} \times 10^{19}$ atom $\mathrm{cm}^{-2}$. The measured temperature of $3.04_{-0.19}^{+0.23} \mathrm{keV}$ is lower than the $5 \mathrm{keV}$ assumed in the simulated spectrum, due to the strong cooling flow. The Abell 1795 spectrum has the same integrated count rate in the $0.1-2.0 \mathrm{keV}$ band as the simulated spectrum. Error bars are $1 \sigma$ confidence limits.

gained from the following argument. We can write the observed flux as

$A(E) e^{-\sigma(E) N_{\mathrm{H}}}\left[(1-f)+f e^{-\sigma(E) \Delta N_{\mathrm{H}}}\right] \approx A(E) e^{-\sigma(E) N_{\mathrm{H}}^{\prime}}$

where $A(E)$ is the emission at energy $E$. Then the apparent column density determined from a fit assuming a covering fraction of unity is

$N_{\mathrm{H}}^{\prime}=N_{\mathrm{H}}-\log \left[1-f+f e^{-\sigma(E) \Delta N_{\mathrm{H}}}\right] / \sigma(E)$,

implying an apparent excess absorption of

$\Delta N_{\mathrm{H}}^{\prime}=-\log \left[1-f+f e^{-\sigma(E) \Delta N_{\mathrm{H}}}\right] / \sigma(E)$.

The absorption cross-section may be crudely approximated by $\sigma(E) \approx 10^{-21}(E / 0.4)^{-8 / 3} \mathrm{~cm}^{-2}$. In Figs. 8(b) and (d) we plot $N_{\mathrm{H}}^{\prime}$ as a function of $f$ for photon energies $E=0.20$ and $0.40 \mathrm{keV}$, and intrinsic column densities of $10^{21}$ and $5 \times 10^{21}$ atom $\mathrm{cm}^{-2}$. (Note that the lowest-energy photons included in any fits will dominate the constraints on the absorption value.) The analytic curves provide a good approximation to the observed behaviour. The apparent excess column density, $\Delta N_{\mathrm{H}}^{\prime}$, remains low so long as significant signal from low energies (below the energy where $\left.\sigma N_{\mathrm{H}} \sim 1\right)$ is included, i.e. $E<0.4 N_{\mathrm{H}, 21}^{3 / 8} \mathrm{keV}$, where $N_{\mathrm{H}}=$ $10^{21} N_{\mathrm{H}, 21} \mathrm{~cm}^{-2}$. Where $\sigma \Delta N_{\mathrm{H}}$ is small, $\Delta N_{\mathrm{H}}^{\prime} \rightarrow f \Delta N_{\mathrm{H}}$.

Our results imply that simple fits to PSPC spectra for clusters with low Galactic column densities $\left(N_{\mathrm{H}} \sim 10^{20}\right.$ atom $\mathrm{cm}^{-2}$ ), which assume covering fractions for the absorber of unity, will always return low values for the excess column density even if, in reality, $f \sim 0.5$ and $\Delta N_{\mathrm{H}} \gtrsim 10^{21}$ atom $\mathrm{cm}^{-2}$. If the true intrinsic column densities in clusters are $\gtrsim 10^{21}$ atom $\mathrm{cm}^{-2}$, we should only obtain reasonable estimates for $\Delta N_{\mathrm{H}}$ with simple spectral fits if we either restrict the bandwidth artificially, or if the cluster has a large Galactic column density $\left(N_{\mathrm{H}} \sim 10^{21}\right.$ atom $\left.\mathrm{cm}^{-2}\right)$. Such a scenario occurred naturally in the PSPC studies of Abell 478 by Allen et al. (1993) and of 2A $0335+096$ by Irwin \& Sarazin (1995), where significant excess column densities of $\sim 10^{21}$ atom $\mathrm{cm}^{-2}$ were inferred.

Such arguments also imply a weak positive correlation between the apparent excess column density, $\Delta N_{\mathrm{H}}^{\prime}$, determined from fits with uniform foreground screen absorption models, and the Galactic column density, $N_{\mathrm{H}}$. When $N_{\mathrm{H}}$ is high then the photon energy dominating the absorption fit (generally the lowest energy with a significant detection) is also high and $\sigma \Delta N_{\mathrm{H}}$ tends to be small, so that the inferred column density $\Delta N_{\mathrm{H}}^{\prime} \approx f \Delta N_{\mathrm{H}}$. Provided that the covering fraction, $f$, is also high, the model will tend to the 'true' answer. This is consistent with the results presented in Table 6, which exhibit a weak correlation between $\Delta N_{\mathrm{H}}^{\prime}$ and $N_{\mathrm{H}}$, particularly once the radial variation of $\Delta N_{\mathrm{H}}^{\prime}$ in Abell 426 is taken into account.

We have used the relationship between $\Delta N_{\mathrm{H}}^{\prime}$ and $\Delta N_{\mathrm{H}}$ in equation 4 , and the results from further spectral simu- 
lations, to estimate the correction factors that should be applied to the $\Delta N_{\mathrm{H}}^{\prime}$ values listed in Table 6 , in determining the true intrinsic column densities in the clusters. For these purposes we assume a covering fraction $f=0.5$ for the intrinsic absorber (which should approximate the true value in the central regions of the clusters). The results are shown in Fig. 10. We see that for an apparent excess column density, $\Delta N_{\mathrm{H}}^{\prime}=2 \times 10^{20}$ atom $\mathrm{cm}^{-2}$, the analytical expression and spectral simulations imply true intrinsic column densities of $4.5 \times 10^{20}$ and $6.1 \times 10^{20}$ atom $\mathrm{cm}^{-2}$, respectively. Typically, the corrected intrinsic column densities for the clusters in our sample are a factor of $2-3$ larger than apparent values determined directly from the B/D analysis. [The corrected intrinsic column densities determined from equation 4 are listed in Column 4 of Table 6 as $\Delta N_{\mathrm{H}}(1)$.]

Finally, it is also important to note that the use of slightly more complex spectral models incorporating both isothermal and cooling-flow components, with the excess absorber modelled as a uniform screen in front of the cooling flow, will still lead to significant underestimates of the true levels of intrinsic absorption in clusters. Typically, only a few per cent of the X-ray emission along the line of sight to the central 30 arcsec bin will be due to material in front of the cooling flows. If the absorbing material is distributed throughout the cooling flow, as required by the observations reported here, any model which places the absorber entirely in front of the cooling flow will underestimate the true intrinsic column densities in much the same manner as in the simulations discussed above.

\subsection{The multilayer absorption model}

We have also explored a second plausible distribution for the X-ray absorbing material in which the absorbing gas is assumed to reside in many small clouds, each of which has an individual column density much less than the total intrinsic column density, $\Delta N_{\mathrm{H}}$. The clouds are assumed to be evenly distributed throughout the X-ray emitting plasma. For an intrinsic emission spectrum $A(E)$, the observed spectrum, $A^{\prime}(E)$, emerging from the emitting/absorbing region may be written as

$A^{\prime}(E)=A(E)\left(\frac{1-e^{-\sigma(E) \Delta N_{\mathrm{H}}}}{\sigma(E) \Delta N_{\mathrm{H}}}\right)$.

A series of spectra incorporating absorption as described by equation 5 (which we hereafter refer to as the 'multilayer' absorption model) were simulated. Again, the intrinsic emission spectrum was taken to be a $5 \mathrm{keV}$ thermal plasma, with a metallicity, $Z=0.5 Z_{\odot}$, and a redshift, $z=0.01$. Total intrinsic column densities of $\Delta N_{\mathrm{H}}=10^{21}$ and $5 \times 10^{21}$ atom $\mathrm{cm}^{-2}$ were examined.

The simulated spectra were then fitted with simple uniform foreground screen absorption models. As with the partial covering model discussed in Section 6.1, the apparent excess column densities determined with the uniform absorbing screen model significantly underestimate the true intrinsic column density. For a Galactic column density, $N_{\mathrm{H}}=10^{20}$ atom $\mathrm{cm}^{-2}$, and intrinsic multilayer column densities of $\Delta N_{\mathrm{H}}=10^{21}$ and $5 \times 10^{21}$ atom $\mathrm{cm}^{-2}$, the apparent excess column densities determined with the uniform absorbing screen model are $\Delta N_{\mathrm{H}}^{\prime}=2.65$ and $10.3 \times 10^{20}$ atom $\mathrm{cm}^{-2}$, respectively. Thus, with a multilayer distribution for the intrinsic absorbing gas, the simple uniform foreground screen model underestimates the true intrinsic column density by factors of $\sim 4$ and 5 respectively. Note also that these factors are essentially independent of the Galactic column density, since multilayer absorption, with total column densities of $\Delta N_{\mathrm{H}}=10^{21}$ and $5 \times 10^{21}$ atom $\mathrm{cm}^{-2}$, can be well-approximated by uniform absorbing screens with column densities of $\sim 0.26$ and $1.0 \times 10^{21}$ atom $\mathrm{cm}^{-2}$, respectively. In Column 5 of Table $6\left[\Delta N_{\mathrm{H}}(2)\right]$, we list the intrinsic column densities for the sample of cooling flows in this paper, corrected (with an intermediate correction factor of 4.5) for an assumed multilayer distribution of the absorbing gas.

\subsection{The comparison of results from ROSAT, SSS and ASCA}

The results presented here confirm that intrinsic X-ray absorption is a common property of cooling flows. All of the clusters with previous positive detections of intrinsic absorption, from the Einstein SSS study of White et al. (1991), also exhibit absorption signatures in their ROSAT X-ray colour profiles. The column densities inferred from the two studies are also similar, ranging from a few $10^{20}$ to a few $10^{21}$ atom $\mathrm{cm}^{-2}$. However, although the results reported here (Table 6 columns 4,5) include corrections for the effects of the likely spatial distribution of absorbing gas on the measured column densities, the White et al. (1991) results were all determined under the more simple assumption that the absorber acts as a uniform screen in front of the clusters, with a covering fraction of unity. When the same simple model for the distribution of the absorbing gas is used with the ROSAT data, (Table 6, Column 3) we obtain results that are typically factors of $2-6$ less than the SSS results. In this Section we examine whether this discrepancy should simply be attributed to calibration uncertainties, or if it instead provides further information about the nature of the absorbing gas.

The SSS observations were made in the $0.6-4.5 \mathrm{keV}$ energy band, with a spectral resolution of $\sim 160 \mathrm{eV}$, a higher energy band and a higher spectral resolution than that of the PSPC. We have examined the variation in the results from fits to simulated cluster spectra, incorporating intrinsic absorption with both partial covering and multilayer distributions, when observed with the two instruments. (With the SSS simulations, the effects of ice build-up on the instrument response were accounted for.) Total multilayer and partial covering column densities of $10^{21}-10^{22}$ atom $\mathrm{cm}^{-2}$ were examined, with a default Galactic column density of $10^{20}$ atom $\mathrm{cm}^{-2}$ assumed. The simulated spectra were then fitted with simple uniform foreground screen models, assuming a covering fraction for the excess absorption of unity.

With a multilayer distribution for the intrinsic absorbing gas in the simulated spectra, the fits with the uniformscreen model give very similar results for the excess column densities with the PSPC and SSS instruments (in both cases the fit results underestimate the true intrinsic column densities by factors of $\sim 4-5 ; c f$. Section 6.2). However, when the intrinsic absorber in the simulated spectra is distributed according to the partial covering model, clear differences between the PSPC and SSS results become apparent.

The differences between the PSPC and SSS results, from the fits to the simulated spectra, incorporating partial 


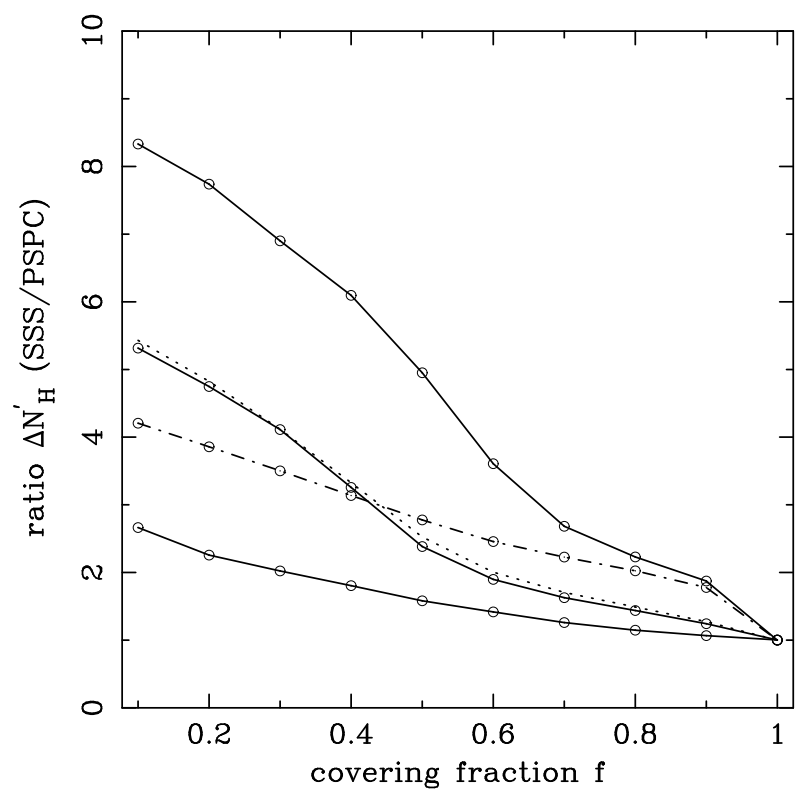

Figure 11. The ratio of the apparent excess column densities, $\Delta N_{\mathrm{H}}^{\prime}$, inferred from fits with simple uniform foreground screen absorption models to simulated SSS and PSPC spectra incorporating intrinsic absorption with a covering fraction, $f$, of between 0.1 and 1.0. The solid curves show the results (from top to bottom) for $\Delta N_{\mathrm{H}}=10,5$ and $1 \times 10^{21}$ atom $\mathrm{cm}^{-2}$, with a fixed Galactic column density, $N_{\mathrm{H}}$, of $10^{20}$ atom $\mathrm{cm}^{-2}$. The dotdashed curve shows the results for $\Delta N_{\mathrm{H}}=10^{22}$ atom $\mathrm{cm}^{-2}$ and $N_{\mathrm{H}}=3 \times 10^{20}$ atom $\mathrm{cm}^{-2}$. The dotted curve shows the ratio of the results for the simulated ASCA SIS and PSPC spectra, for $\Delta N_{\mathrm{H}}=5 \times 10^{21}$ atom $\mathrm{cm}^{-2}$ and $N_{\mathrm{H}}=10^{20}$ atom $\mathrm{cm}^{-2}$. Note the agreement between the SIS/PSPC and SSS/PSPC results.

covering, are summarized in Fig. 11. For a Galactic column density of $\sim 10^{20}$ atom $\mathrm{cm}^{-2}$, and intrinsic absorption with a column density of a few $10^{21}$ atom $\mathrm{cm}^{-2}$ and a covering fraction $f=0.2-0.6$, discrepancies of factors of between 2 and 6 will naturally arise between measurements made with the SSS and PSPC instruments (in the sense the SSS results exceed the PSPC results). Such an effect is sufficient to account for the bulk of the discrepancy between the White et al. (1991) SSS results and the ROSAT results reported here.

We have also carried out a further comparison, between the PSPC results and the results from simulated spectra generated with an instrument response appropriate for the Solid-state Imaging Spectrometers (SISs) on ASCA. The dotted curve in Fig. 11 shows the ratio of the excess column density determined from the ASCA SIS simulations to that with ROSAT PSPC, for a Galactic column density $N_{\mathrm{H}}=10^{20}$ atom $\mathrm{cm}^{-2}$ and an intrinsic column density of $5 \times 10^{21}$ atom $\mathrm{cm}^{-2}$. The results are very similar to those obtained from the comparison of the SSS and PSPC data, for the same absorption parameters.

Since the partial covering model can account for many of the observed properties of the intrinsic absorber, we have made one further check on the validity of this model by applying it to observed ROSAT PSPC, Einstein SSS and ASCA SIS spectra for Abell 2199 (which has the best com- bined data set of any of the clusters in the present sample). Spectra from the central 2 arcmin (radius) region of Abell 2199 were extracted from the PSPC and ASCA SIS data sets ( 2 arcmin radius being the smallest region that can be consistently analysed with the two instruments, given the relevant PSFs). The SSS offers no spatial resolution but did have a 3 arcmin (radius) circular aperture, reasonably wellmatched to the regions studied with the other instruments. With the ASCA analysis, the data from both SIS detectors were used. With the SSS analysis, the data from all three observations of the source (White et al. 1991) were included.

The spectra were fitted with a model incorporating emission from an isothermal plasma and a cooling flow of $80 \mathrm{M}_{\odot} \mathrm{yr}^{-1}$ (cf. Section 5.5), at a redshift of $z=0.0309$, absorbed by a (fixed) Galactic column density of $8.7 \times 10^{19}$ atom $\mathrm{cm}^{-2}$ (except for the PSPC data where the Galactic column density was fixed at $5 \times 10^{19}$ atom $\mathrm{cm}^{-2}$; see Section 5.3) . A second absorption component of variable column density and covering fraction was also assumed to act on the emission. The cooling flow was forced to cool from the ambient cluster temperature, which was a free parameter in the fits (with the exception of the ROSAT analysis where, due to the limitations of the data, the upper temperature of the flow was fixed at $3.5 \mathrm{keV}$, in agreement with the results of Section 5). The metallicity of the X-ray emitting gas and the emission measure of the isothermal component were also free parameters in the fits. With the ROSAT data, the spectral analysis was limited to the $0.4-2.0 \mathrm{keV}$ energy range. For the ASCA SIS and Einstein SSS data, spectral ranges of $0.6-10.0 \mathrm{keV}$ and $0.6-4.0 \mathrm{keV}$ were used, respectively. All appropriate ice corrections were applied to the SSS data.

The joint confidence contours on the column density and covering fraction of the excess absorber in Abell 2199 are shown in Fig. 12. The contours indicate the regions of 68, 90 and 99 per cent confidence on two interesting parameters. The SIS and SSS data, in particular, exhibit good agreement. For the SIS data, the best-fit excess column density is $2.5_{-1.0}^{+1.1} \times 10^{21}$ atom $\mathrm{cm}^{-2}$ and the covering fraction, $f=0.53_{-0.07}^{+0.17}$. For the PSPC data, the best-fit values are $\Delta N_{\mathrm{H}}=1.2_{-0.3}^{+0.7} \times 10^{21}$ atom $\mathrm{cm}^{-2}$ and $f=0.43_{-0.13}^{+0.14}$, where errors are the 90 per cent $\left(\Delta \chi^{2}=2.71\right)$ confidence limits on a single interesting parameter.

The simple partial covering model thus provides a reasonable description of the excess absorption in Abell 2199 and leads to reasonable agreement in the parameter values inferred from the different data sets. However, although the errors on the individual parameter values overlap, the $\Delta \chi^{2}$ contours shown in Fig. 12 indicate an inconsistency between the PSPC data and the other two data sets. This implies either a deficiency in the spectral model or a calibration problem with the instruments. The simple partial-covering model used undoubtedly underestimates the complexity of the absorption in a real cluster, where the column density acting on the emission from different regions is likely to span a range of values. The abundances of separate elements in the absorber could also deviate from the assumed solar values, perhaps as a function of radius. Further complexities would be introduced if the absorbing material were dusty, which recent evidence suggests is likely to be the case (Fabian, Johnstone \& Daines, Allen et al. 1995, Voit \& Donahue 1995, Allen et al. 1996). If, however, the origin of the discrepancy is a calibration problem, the agreement between the SIS and SSS 


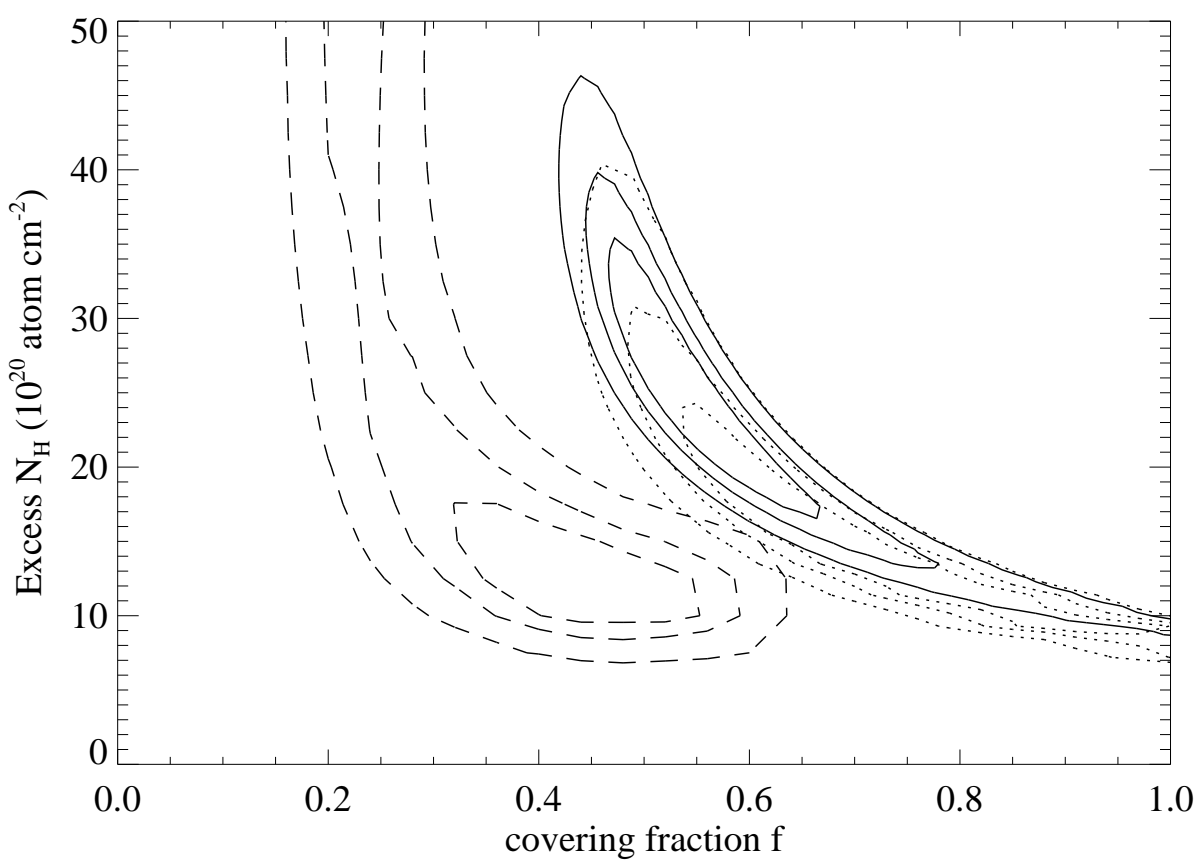

Figure 12. The joint confidence contours on the column density and covering fraction of the intrinsic absorber in Abell 2199 for the ASCA SIS (solid curves), ROSAT PSPC (dashed curves) and Einstein SSS (dotted curves) data. The contours mark the $\Delta \chi^{2}=2.30,4.61$ and 9.21 limits, corresponding to 68, 90 and 99 per cent confidence for two interesting parameters.

results would suggest that the problem is likely to lie with the PSPC data.

\subsection{The mass of absorbing gas}

The results on the intrinsic column densities, summarized in Table 6 , allow a simple estimation of the mass of absorbing gas in the central 30 arcsec (radius) regions of the clusters to be made. Adopting the partial covering model for the distribution of the absorbing gas, with $f=0.5$, and assuming solar metallicity in this material, the mass of gas can be written as $M_{\text {abs }}=1.62 \times 10^{6} r^{2} \Delta N_{\mathrm{H}, 20} \mathrm{M}_{\odot}$, where $r$ is the radius of the 30 arcsec region in $\mathrm{kpc}$ and $\Delta N_{\mathrm{H}, 20}$ is the intrinsic column density in units of $10^{20}$ atom $\mathrm{cm}^{-2}$. We can also estimate the time, $t_{\text {acc }}$, required for the cooling flows to accumulate these masses of gas, given the observed mass deposition rates in these regions $\left(t_{\mathrm{acc}}=M_{\mathrm{abs}} / \dot{M}\right)$. [Note that the mass of absorbing gas is actually a projected mass through the central 30 arcsec region, whereas the mass deposition rates apply to the central 30 arcsec volume. Thus, where significant mass deposition occurs outside the central 30 arcsec region, the accumulation timescales, $t_{\text {acc }}$, quoted will slightly overestimate the true values. Assuming $\dot{M} \propto r$, the projected mass deposition rate through the central 30 arcsec region will be $\sim 30$ per cent higher than the rate within that volume, and the accumulation timescale will be $\sim 25$ per cent shorter than the quoted result.] The masses of absorbing gas, mass deposition rates, accumulation times, and the masses of hot X-ray emitting gas within the central 30 arcsec (radius) volumes, are summarized in Table 7.

From Table 7 we see that the accumulation timescales are typically only a few $10^{8}$ years, much less than the ages of the cooling flows ( $c f$. Section 5.5.) This implies that most of the mass deposited by the cooling flows in the central regions of the clusters must reside in some form other than X-ray absorbing gas (perhaps low mass stars or brown dwarfs; Fabian 1994). Note, however, that if the metallicity of the absorbing material is substantially sub-solar, the true masses of absorbing gas are likely to be $\sim 2-3$ times larger than the values listed in Table 7 . Note also that the mass of absorbing material (assuming solar metallicity) is typically $\lesssim 10$ per cent of the mass of the X-ray emitting gas in the same regions (although for Abell 478 and MKW3s the values are somewhat higher.)

\section{CONCLUSIONS}

In this paper we have described and discussed the results from a detailed X-ray colour profile analysis of a sample of nearby, luminous cooling flow clusters observed with the ROSAT PSPC. Our analysis of the C/D (0.80 - 1.39 $\mathrm{keV} / 1.40-2.00 \mathrm{keV})$ ratios clearly demonstrates the presence of distributed cool gas in the central regions $\left(r \lesssim r_{\text {cool }}\right)$ of the cooling flow clusters. The spatial distributions and emissivity of the cooling gas are in excellent agreement with the predictions from standard image deprojection analyses. In the outer regions of the clusters the data suggest approximate isothermality. Our results place new constraints on the ages of the cooling flows and provide the most accurate determinations of the mass deposition rates in these systems of any imaging study to date.

The analysis of the B/D $(0.41-0.79 \mathrm{keV} / 1.40-2.00$ $\mathrm{keV}$ ) ratios provides firm evidence for spatially distributed 

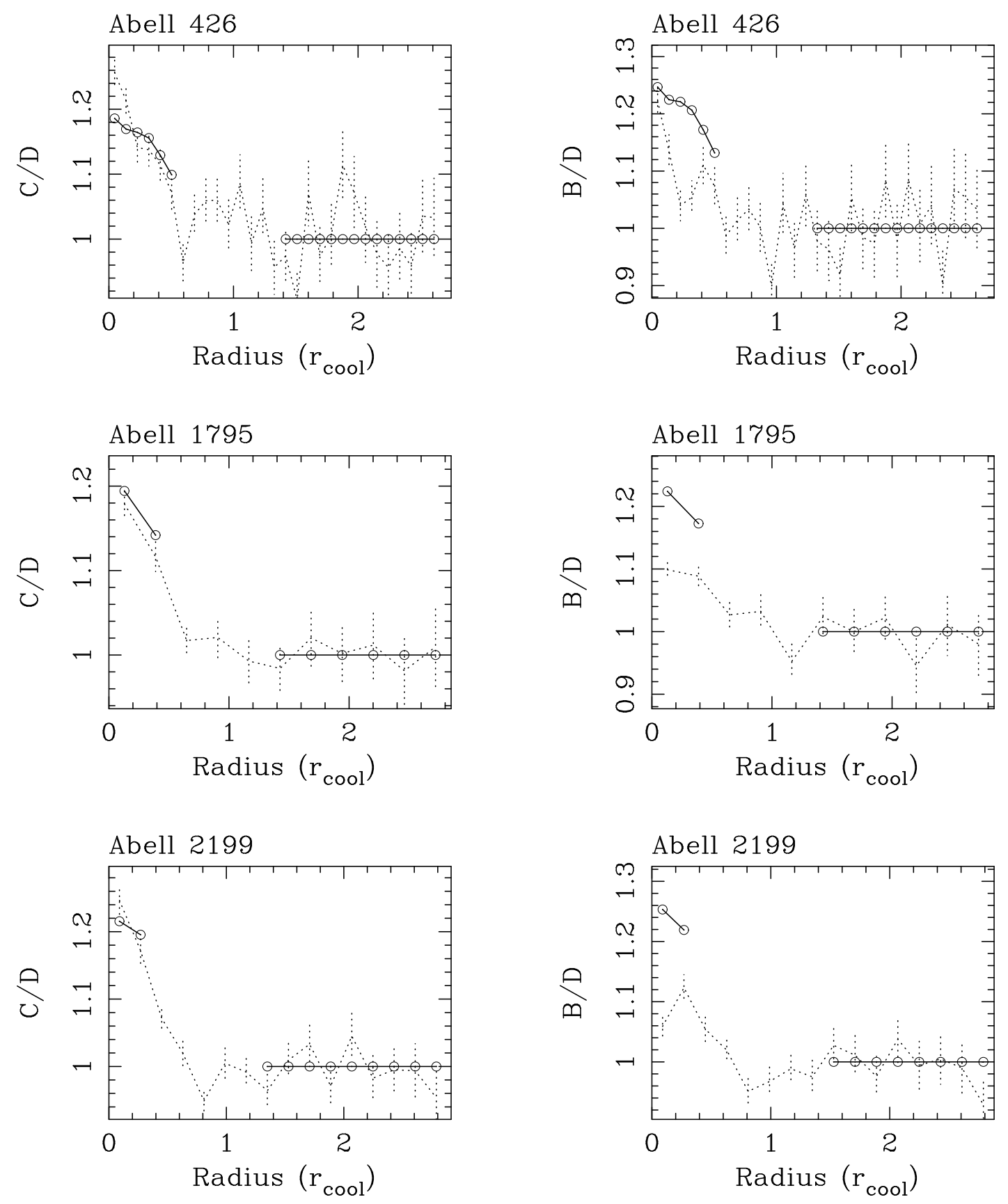

Figure 13. A summary of the results on the distributions of cool gas and intrinsic absorbing material in Abell 426 , Abell 1795 and Abell 2199. The y axes show the $\mathrm{C} / \mathrm{D}$ and $\mathrm{B} / \mathrm{D}$ ratios normalized to the values at large radii (Table 5). The $\mathrm{x}$ axes are in units of the cooling radii, $r_{\text {cool }}$ (see Table 3 ). The C/D profiles clearly demonstrate the presence of distributed cool gas within the cooling radii, and the $\mathrm{B} / \mathrm{D}$ data the need for excess absorption in the central regions. Other details as in Figs. 3 and 5. 
Table 7. The masses of absorbing gas and accumulation timescales

\begin{tabular}{ccccc} 
Cluster & $\begin{array}{c}M_{\text {abs }} \\
\left(10^{9} \mathrm{M}_{\odot}\right)\end{array}$ & $\begin{array}{c}\dot{M} \\
\left(\mathrm{M}_{\odot} \mathrm{yr}^{-1}\right)\end{array}$ & $\begin{array}{c}t_{\mathrm{acc}} \\
\left(10^{8} \mathrm{yr}\right)\end{array}$ & $\begin{array}{c}M_{\text {hot }} \\
\left(10^{10} \mathrm{M}_{\odot}\right)\end{array}$ \\
\hline & & & & \\
Abell 85 & $15.0_{-4.4}^{+4.7}$ & $36.3_{-1.9}^{+1.4}$ & $4.1_{-1.1}^{+1.3}$ & $20.9 \pm 0.4$ \\
Abell 426 & $0.4_{-0.4}^{+0.5}$ & $16.0_{-1.1}^{+0.9}$ & $0.25_{-0.25}^{+0.31}$ & $3.0 \pm 0.1$ \\
Abell 478 & $143_{-17}^{+19}$ & $160_{-5}^{+4}$ & $8.9_{-1.1}^{+1.2}$ & $88.6 \pm 1.0$ \\
Abell 496 & $6.8_{-1.8}^{+2.2}$ & $17.2_{-0.7}^{+0.9}$ & $4.0_{-1.1}^{+1.3}$ & $6.8 \pm 0.1$ \\
Hydra A & $21.1_{-3.4}^{+3.9}$ & $69.4_{-1.2}^{+1.7}$ & $3.0_{-0.5}^{+0.6}$ & $25.9 \pm 0.3$ \\
Abell 1795 & $20.0_{-2.0}^{+2.4}$ & $83.4_{-2.1}^{+2.0}$ & $2.4_{-0.2}^{+0.3}$ & $35.7 \pm 0.3$ \\
Abell 2029 & $9.5_{-5.1}^{+5.6}$ & $108_{-4.4}^{+3.4}$ & $0.88_{-0.47}^{+0.52}$ & $57.7 \pm 0.9$ \\
MKW3s & $34.7_{-5.1}^{+5.6}$ & $19.7_{-1.4}^{+1.4}$ & $17.6_{-2.6}^{+2.9}$ & $9.0 \pm 0.3$ \\
Abell 2199 & $6.7_{-0.7}^{+0.8}$ & $13.6_{-0.3}^{+0.4}$ & $4.9_{-0.5}^{+0.6}$ & $5.6 \pm 0.1$ \\
Cygnus A & $44_{-10}^{+11}$ & $118_{-5}^{+5}$ & $3.7_{-0.8}^{+0.9}$ & $39.3 \pm 0.6$ \\
Sersic 159 & $13.3_{-3.8}^{+3.8}$ & $61.6_{-1.8}^{+2.3}$ & $2.2_{-0.6}^{+0.6}$ & $24.6 \pm 0.3$ \\
Abell 2597 & $35_{-10}^{+10}$ & $126_{-5}^{+4}$ & $2.8_{-0.8}^{+0.8}$ & $72.5 \pm 1.1$
\end{tabular}

The masses of absorbing gas, $M_{\mathrm{abs}}$ (calculated assuming solar metallicity in the absorbing material), mass deposition rates, $\dot{M}$, accumulation timescales, $t_{\mathrm{acc}}$, and masses of hot, X-ray emitting gas, $M_{\mathrm{hot}}$, in the central $30 \operatorname{arcsec}$ regions of the clusters. The $M_{\mathrm{abs}}$ results are derived from the $\left[\Delta N_{\mathrm{H}}(1)\right]$ column density measurements made with the partial covering model (Table 6, Column 4). A covering fraction $f=0.5$ has been assumed for all clusters except MKW3s, for which $f=0.75$ was used. The $\dot{M}$ and $M_{\text {hot }}$ values apply to the central 30 arcsec (radius) volume.

intrinsic absorbing material in the cooling flows. The absorbing gas generally exhibits an increasing column density with decreasing radius, and is confined to radii $\lesssim r_{\text {cool }}$. Our results are consistent with the large intrinsic column densities $\left(\Delta N_{\mathrm{H}} \sim 10^{21}\right.$ atom $\left.\mathrm{cm}^{-2}\right)$ inferred from previous studies with the Einstein Observatory SSS and ASCA (White et al. 1991; Fabian et al. 1994) but require that the absorber only partially covers the emitting region. This suggests significant clumping of the absorber on large scales.

We have discussed the complexities involved in the measurement of intrinsic column densities in clusters and have shown that the use of simple spectral models, which treat the absorber as a uniform foreground screen (in front of the cooling flow or cluster) will naturally lead to significant underestimates (by factors of $\sim 2-5$ ) of the true amounts of absorbing material.

Assuming solar metallicity in the absorbing material, the masses of absorbing gas in the central 30 arcsec regions of the clusters typically correspond to $\lesssim 10$ per cent of the mass of the hot, X-ray emitting gas. The timescales required for the cooling flows to accumulate such masses are significantly shorter than ( $\sim 10$ per cent of) the likely ages of the cooling flows, and imply that the bulk of the matter deposited by the cooling flows (in the central 30 arcsec radius regions) resides in some form other than X-ray absorbing gas, presumably stars or sub-stellar objects.

The new results presented in this paper provide strong support for the standard inhomogeneous model of cooling flows in clusters of galaxies. Future studies of the range of temperature/density phases in cooling flows (as a function of radius), more precise determinations of the 'ages' of cooling flows, and more detailed mapping of the intrinsic column densities in clusters, will become possible following the launch of AXAF.

\section{ACKNOWLEDGMENTS}

We thank Roderick Johnstone and Dave White for software assistance, and the Royal Society for support.

\section{REFERENCES}

Allen S.W., 1995, MNRAS, 276, 947

Allen S.W., Fabian A.C., 1994, MNRAS, 269, 409

Allen S.W., Fabian A.C., Kneib J.P., 1996a, MNRAS, 279, 615

Allen S.W., Fabian A.C., Johnstone R.M., Nulsen P.E.J., Edge A.C., 1992, MNRAS, 254, 51

Allen S.W., Fabian A.C., Johnstone R.M., White D.A., Daines S.J., Edge A.C., Stewart G.C., 1993, MNRAS, 262, 901

Allen S.W., Fabian A.C., Edge A.C., Böhringer H., White D.A., 1995, MNRAS, 275, 741

Allen S.W., Fabian A.C., Edge, A.C., Bautz M.W., Furuzawa A., Tawara Y., 1996, MNRAS, in press

Anders E., Grevesse N., 1989, Geochemica et Cosmochimica Acta 53, 197

Böhringer H., Voges W., Fabian A.C., Edge A.C., Neumann D.M., 1993, MNRAS, 264, L25

Crawford C.S., Fabian A.C., 1992, MNRAS, 259, 265

Cowie L.L., Binney J., 1977, ApJ, 215, 723

Edge A.C., Stewart G.C., Fabian A.C., Arnaud K.A., 1990, MNRAS, 245,559

Edge A.C., Stewart G.C., Fabian A.C., 1992, MNRAS, 258, 177

Fabian A.C., 1994, A\&AR, 32, 277

Fabian A.C., Nulsen P.E.J., 1977, MNRAS, 180, 479

Fabian A.C., Nulsen P.E.J., Canizares C.R., 1991, A\&AR, 2, 191

Fabian A.C., Johnstone R.M., Daines S.J., 1994, MNRAS, 271, 737

Fabian A.C., Hu E.M., Cowie L.L., Grindlay J., 1981, ApJ, 248, 47

Fabian A.C., Arnaud K.A., Bautz M.W., Tawara Y., 1994, ApJ, 436, L63

Fabian A.C. et al. 1996, in preparation 
Fukazawa Y. et al. 1994, PASJ, 46, L55

Hasinger G., Turner T.J., George I.M., Boese G., 1992, OGIP Calibration Memo CAL/ROS/92-001, NASA

Hu E.M., Cowie L.L., Wang Z., 1985, ApJS, 59, 447

Irwin J.A., Sarazin C.L., 1995, ApJ, 355, 497

Johnstone R.M., Fabian A.C., Nulsen P.E.J., 1987, MNRAS, 224, 75

Johnstone R.M., Fabian A.C., Edge A.C., Thomas P.A., 1992, MNRAS, 255, 431

Kaastra J.S., Mewe R., 1993, Legacy, 3, HEASARC, NASA

Mathews W.G., Bregman J.N., 1978, ApJ, 244, 308

Morrison R., McCammon D.M., 1983, ApJ, 270, 119

Ohashi T. et al. 1996, in preparation

Shafer R.A., Haberl F., Arnaud K.A., Tennant A.F., 1991, XSPEC User's Guide,. ESA, Noordwijk

Stark A.A., Gammie C.F., Wilson R.W., Bally J., Linke R.A., Heiles C. \& Hurwitz M., 1992. ApJS, 79, 77

Voit G.M., Donahue M., 1995, ApJ, 452, 164

Thomas P.A., Fabian A.C., Nulsen P.E.J, 1987, MNRAS, 228, 973

White D.A., Fabian A.C., Johnstone R.M., Mushotzky R.F., Arnaud K.A., 1991, MNRAS, 252, 72 